This report was prepared as an account of work spunsored by an agency of the United States Government. Neither the United States Government nor any agency thereof, nor any of their emptoyees. maka any wartanty. express or implied, or assuries any legal "ability or responsi. bility for the accurac, compieteness of usefulness of any information, appatatus. pruduct. or process diselosed. or represents that its use would not infringe privately owned rights. Reference trerein to any specific commereial produsi protes. or service by irade name. irademark. manufacturet or otherwise does nor nevessarily zonstinte or imply its endorsemeni, tecom. mendation. or favoring by the Lnited States Government or any agensy thereaf. The views and opinions of authors expressed terein do not necexuriiy siate of reflect inose of the Unied States Government or any azeno thereci

\title{
Comparative Analyses of Spent Nuclear Fuel Transport Modal Options: Transport Options Under Existing Site Constraints
}

\author{
Technical Report
}

August 1989

2

\author{
L. A. Brentlinger \\ P. L. Hoimann \\ R. W. Peterson*
}

- Currently with Science Applications International Corporation.

Oíice of Transportation Systems and Planning

Battelle Nuclear Systems Group

$505 \mathrm{King}$ Avenue

This document is

Columbus, $\mathrm{OH} 43201-2693$

PUBLICLY RELEASABLE

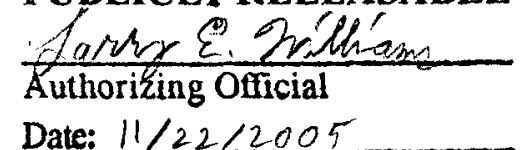

Date: $11 / 22 / 2005$

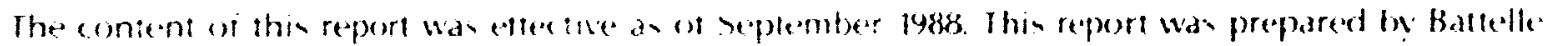

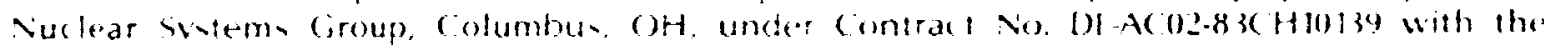
lis. Deparment of fnerat: 


\section{DISCLAIMER}

This report was prepared as an account of work sponsored by an agency of the United States Government. Neither the United States Government nor any agency Thereof, nor any of their employees, makes any warranty, express or implied, or assumes any legal liability or responsibility for the accuracy, completeness, or usefulness of any information, apparatus, product, or process disclosed, or represents that its use would not infringe privately owned rights. Reference herein to any specific commercial product, process, or service by trade name, trademark, manufacturer, or otherwise does not necessarily constitute or imply its endorsement, recommendation, or favoring by the United States Government or any agency thereof. The views and opinions of authors expressed herein do not necessarily state or reflect those of the United States Government or any agency thereof. 


\section{DISCLAIMER}

Portions of this document may be illegible in electronic image products. Images are produced from the best available original document. 
ABSTRACT

The movement of nuclear waste can be accomplished by various transport modal options involving different types of vehicles, transport casks, transport routes, and intermediate intermodal transfer facilities. A series of systems studies are required to evaluate modal/intermodal spent fuel transportation options in a consistent fashion.

This report provides total life-cycle cost and life-cycle dose estimates for a series of transport modal options under existing site constraints.

ii i 
The National waste Terminal Storage Program was established in 1976 by the U.S. Department of Energy's predecessor, the Energy Research and Development Administration. In September 1983, this prcgram became the Civilian Radioactive Waste Management (CRiw) Program. Its purpose is to develop technology and provide facilities for safe, environmentally acceptable, permanent disposal of high-level waste (HLW). HLW includes wastes from both commercial and defense sources, such as spent (used) fuel from nuclear power reactors, accumulations of wastes from production of nuclear weapons, and solidified wastes from fuel reprocessing.

The information in this report covers nuclear waste transport modal option studies for the CRiNM Program. 
Life-cycle cost and life-cycle dose estimates have been made for a series of transport modal options pertaining to the "No-New Orders-'86" nuclear waste scenario. The NNO-'86 scenario encompasses 125 reactor plants and waste poo!s.

Based on current cost data, the findings indicate that truck transport is considerably more expensive than rai? transport. An "all legal-weight truck" case was estimated to cost 2.1 billion dollars $(\$ 24 / \mathrm{kg})$. An "all-rail" case, involving several intermodal transfers, was costed at 1.5 billion dollars $(517 / \mathrm{kg})$.

The lowest cost was obcained for a case using legal-weight (LWiT), rail, and overweight truck (OWT) casks to the extent that crane capacity at the ruciear plants is adequate. The cost for this case was 1.3 billion dollars $(\$ 14 / \mathrm{kg})$. Until OWT permit issues are resolved, the results of this case must be considered as a hypothetical construct only.

Total life-cycle doses range from a high 1.0 person-rem/MTU for the LWT case to 0.18 person-rem/MTU for the rail case. Public doses are considerably less. 
Page

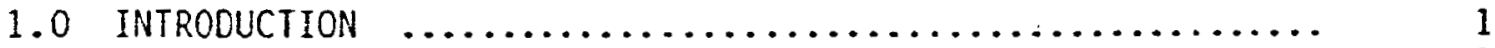

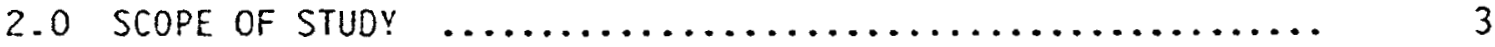

2.1 COMPONENTS OF THE WASTE TRANSPORT SYSTEM $\ldots \ldots \ldots \ldots \ldots \ldots$

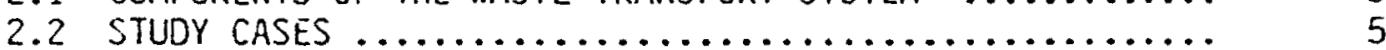

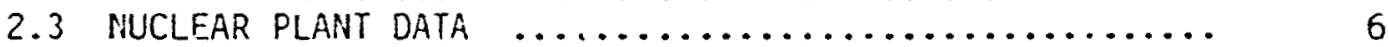

3.0 STUDY BASES AND ASSUMPTIONS $\ldots \ldots \ldots \ldots \ldots \ldots \ldots \ldots \ldots \ldots \ldots \ldots \ldots \ldots \ldots$

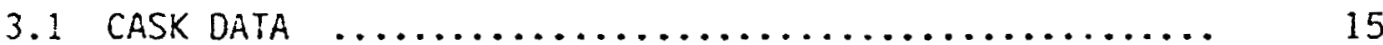

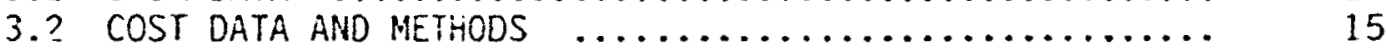

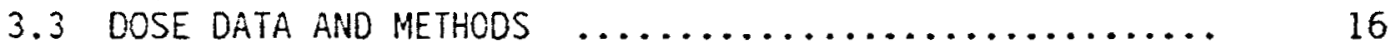

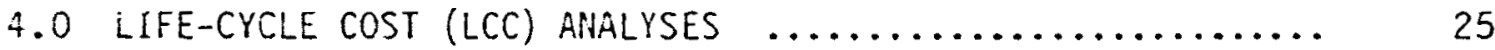

5.0 LIFE-CYCLE DOSE (LCO) ANALYSES $\ldots \ldots \ldots \ldots \ldots \ldots \ldots \ldots \ldots \ldots \ldots$

6.0 SUMMARY AND CONCLUSIONS $\ldots \ldots \ldots \ldots \ldots \ldots \ldots \ldots \ldots \ldots \ldots \ldots \ldots \ldots$

7.0 REFERENCES $\ldots \ldots \ldots \ldots \ldots \ldots \ldots \ldots \ldots \ldots \ldots \ldots \ldots \ldots \ldots \ldots \ldots \ldots \ldots \ldots \ldots \ldots$

APPENDIX A: LCC MODEL DESCRIPTION .................... 53

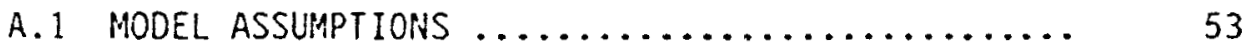

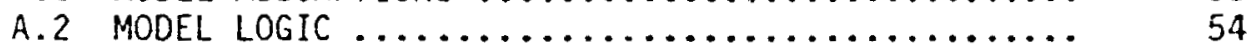




\section{LIST OF TABLES}

Table

Title

Page

2-1. Detailed Reactor/Storage Pool Data for the

Transport Modai Options Study ...................... 8

2-2. Aggregated Reactor!Storage Pool Data for the

Transport Modal Options Study ....................... 13

3-i. Reference Cask Designs for the Transport

Modal Options Study ............................... 15

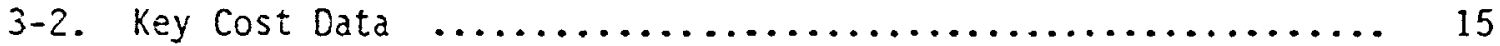

3-3. Estimated Costs for iruck/Rail LO/LO Cask Transfer ......... 17

3-4. Estimated Costs for HHT/Rail RO/RO Cask Transfer ......... 18

3-5. Fopulation Dose Summaries for Spent Fuel

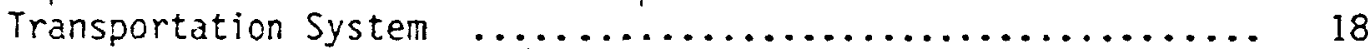

3-6. Collective Dose Data - Truck/Rail LO/LO Transfer .......... 19

3-7. Collective Dose Data - HHT/Rail RO/RO Transfer ........... 24

4-1. Case I. AlT Casks Shipped by LWT $\ldots \ldots \ldots \ldots \ldots \ldots \ldots \ldots \ldots . \ldots . \ldots . \ldots$

4-2. Case II. Reference Case: LWT/RR ................... 28

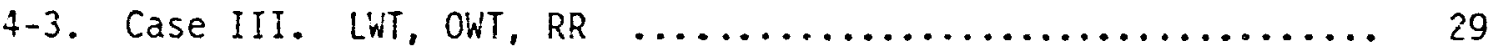

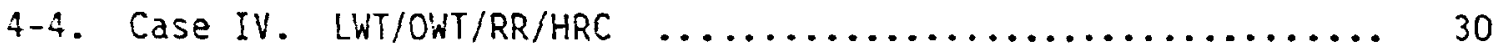

4-5. Case V. LWT/ONT[R]/RR/HRC - Intermodal Transport $\ldots \ldots \ldots \ldots 31$

4-6. Case VI. LWT[R]/OWT[R]/RR/HRC - Intermodal Transport ..... 32

4-7. Case VIIa; LWT/RES. OWT/HHI; $\mathrm{R} / \mathrm{RR} / \mathrm{HRC}-$

4-8. Case VIIb. LWT/RES. OWT[R]/HHT [R]/RR/HRC -

Intermodal Transport $\ldots \ldots \ldots \ldots \ldots \ldots \ldots \ldots \ldots \ldots \ldots \ldots . \ldots \ldots \ldots$

4-9. Case VIIC. LWT[R]/RES. OWT[R]/HHT[R]/RR/HRC -

Intermodal Transport

5-1. LCO Summary - Case I

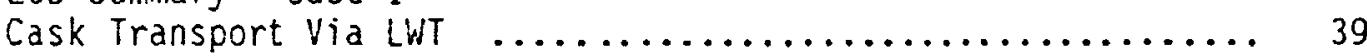

5-2. LCD Sumnary - Case II

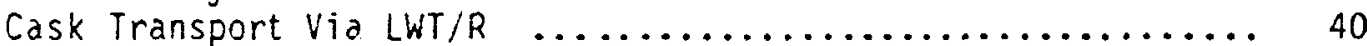


LIST OF TABLES

(Continued)

Table

Title

$\underline{\text { Page }}$

5-3. LCD Summary - Case III

Cask Transport Via OWT/LWT/R

41

5-4. LCD Summary - Case IV

Cask Transport Via O'T/L'T/R/HRC

42

5-5. LCD Summary - Case V

Cask Transport Via OWT(R)/LWT/R/HRC

43

5-6. LCD Summary - Case VI

Sask Transport Via CWT(R)/LWT (R)/R/HRC $\ldots \ldots \ldots \ldots \ldots \ldots \ldots \ldots . \ldots 4$

5-7. LCD Summary - Case VII

Cask Transport Via HHT(R)/OWT/LWT/R/HRC

45

5-8. LCD Summary - Case VII

Cask Transport Via HHT $(R) / O W T(R) / L W T / R / H R C \quad \ldots \ldots \ldots \ldots \ldots \ldots . .46$

5-9. LCD Summary - Case VIIC

Cask Transport Via HHT(R)/OWT(R)/LWT(R)/R/HRC

47

6-1. Summary LCC/LCD Estimates

49

LIST OF FIGURES

Figure

Title

Page

2-1. Transport Modes

5

3-1. Truck-to-Rail Cask Transfer Operations (LO/LO) ........... 20

3-2. HHT Transporter and Auxiliary Equipment [Based on Reference (11)]

21

3-3. RO/RO Operations [Based on Reference (ii)] ............ 22

3-4. HHT-to-Rail Cask transfer Operations (RO/RO) $\ldots \ldots \ldots \ldots \ldots . .23$

4-1. Cost Dependence on Casks/Train

26

6-1. LCC/LCD Summary

50

xi xii 


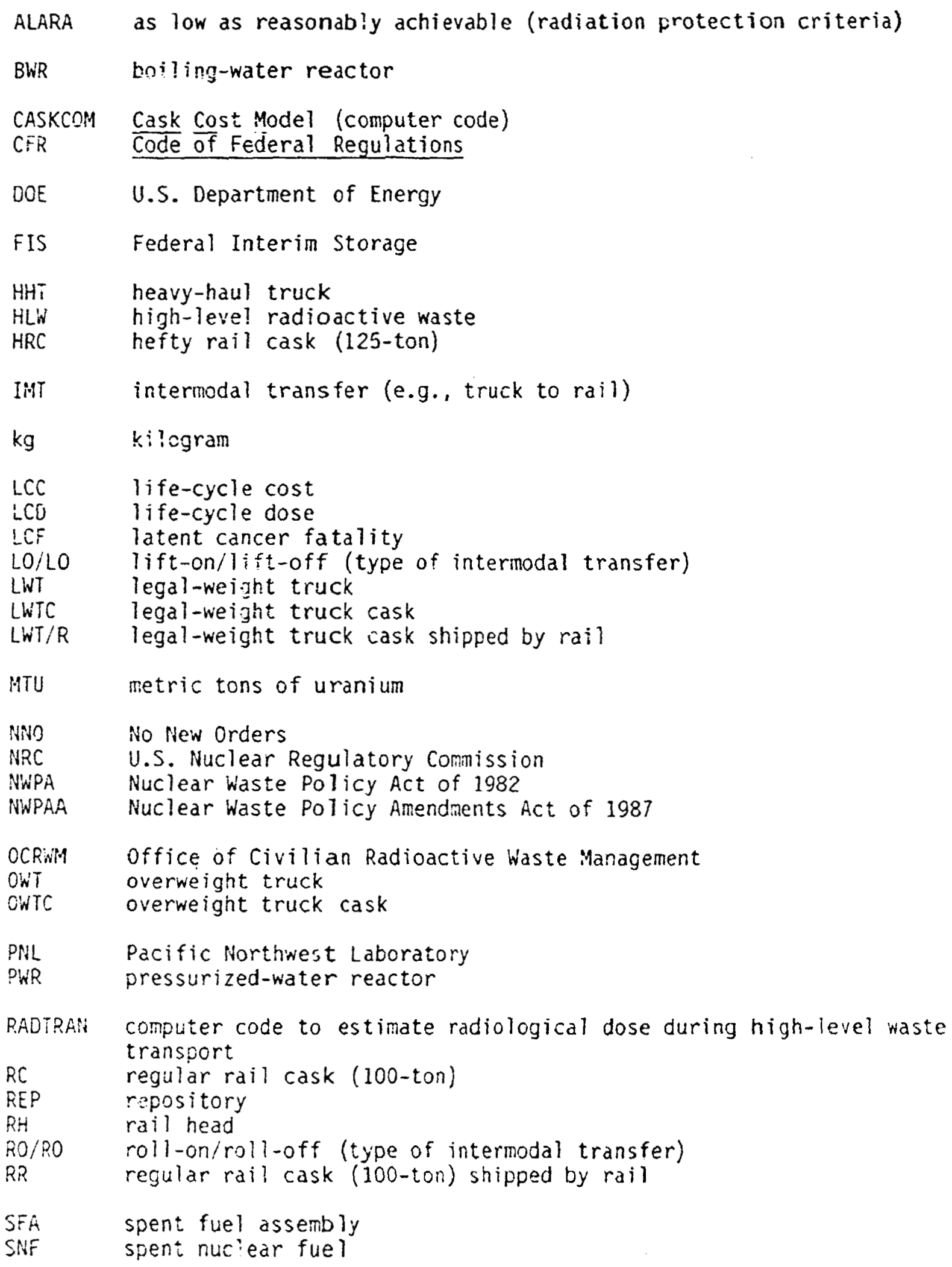


In the Nuclear Waste Policy Act (NWPA) of 1982 (P.L. 97-425), the U.S. Department of Energy (DOE) was given the responsibility for managing the disposal of spent nuclear fuel (SNF) and high-level radioactive waste (HLW) from civilian sources. The DOE Office of Civilian Radioactive Waste Management (OCRWM) was established for that purpose. In December 1987, Congress amended the NWPA by passing the Nuclear Waste Policy Amendments Act (NWPAA) of 1987 (P.L. 100-203). This act was signed into law on December 22, 1987. These two acts currently govern HLW disposal in the United States. Some parts of these acts pertain to the waste transportation system which handles the movement of wastes from designated points of origin either directly, or indirectiy via intermediate facilities, to the final waste repository.

The transportation casks are to be developed by private industry and to be certified by the U.S. Nuclear Regulatory Comission (NRC). The capacities of new casks that are to be developed differ considerably from the casks in current usage. Because the SNF to be shipped in the future will be substantially cooler and less radioactive than most fuel shipped to date, cask capacities are expected to be larger than the capacities of the current casks. Also, if fuel elements were to be consolidated prior to shipment, cask capacities would differ from the current versions.

The movement of nuclear waste could be accomplished by various transport modal options involving different types of vehicles, transport casks, transport routes, and intermediate intermodal transfer facilities. A series of systems studies are required to evaluate modal/intermodal spent fuel transportation options in a consistent fashion.

As indicated in the Iransportation Institutional Plan (1), the modal/ intermodal spent fuel transportation mix is an important transportation issue requiring that "OCRiM continue to analyze . . modes of transport including relative safety, efficiency, capital costs, hauling costs,. . . shipping and receiving facility operations, (and) environmental impacts." Similarly, the OCRim Systems Engineering Management Plan (2), specifies that system studies are necessary "to support the integration, evaluation, and optimization of the system." Finally, 10 CFR Part 961 (Standard Contract for Disposal of Spent Nuclear FuE? and/or High-Level Radioactive Waste) calls for DOE to prepare a reactor-specific service plan by early calendar year 1992, which includes specification of the service mode (3).

In the current study a comparative analysis of only a subset of feasible transport modal options is made. Specifically, the study is confined to the movement of SNF via rail and/or truck in the most direct way from the nuclear reactor plants to the Nevada Yucca Mountain site.* The analyses are further restricted to transport operations under existing site constraints, primarily related to cask handling capabilities and transport access. A no-MRS system is considered, i.e., no additional capital investments are required. Future studies will examine options that do require sizeable capital outlays for atreactor site/facilities and intermodal transfer facilities.

*The Yucca Mountain site has been designated by Congress for characterization to determine its suitability as a location for a high-level nuclear waste repository. 
The comparative analyses address two aspects of the problem: risk and cost. Risk is discussed from the viewpoint of life-cycle dose (LCD) to workers and the public under normal, non-accident operating conditions. Cosis are also determined and compared on an overall life-cycle basis. Cosis and risis of accidents have not been included in this preliminary assessment of modal options, because the radiological risks of such accidents are extremely low and would not affect the choices of modal transport options, especially at reactor sites. Accident analyses have been extensively addressed in other studies.

In the following sections, the scope of the current study is discussed, the bases and assumptions of the study are identified, and finally, the LCD and life-cycle cost (LCC) results for various transport modal options are given and compared. 


\subsection{SCOPE OF STUDY}

\subsection{COMPONENTS OF THE WASTE TRANSPORT SYSTEM}

To carry out the comparative analyses of the set of faacible transport modal/intermodal options, it is convenient to think of the waste transport system as consisting of essentially four kinds of elements:

- Vehicles,

- Casks,

- Facilities, and

- Routes.

A modal/intermodal option can then be defined as a specific combination of these elements.

The vehicle category consists of trucks (three types), railcars, and barges, i.e.,
- Legal-weight trucks,
- Overweight trucks,
- Heavy-haul trucks,
- Railcars, and
- Barges.

The casks that generally go with these vehicles are:

- Legal-weight truck casks,

- Overweight truck casks,

- Regular rail casks,

- Hefty rail casks,

- Transportable storage casks, and

- Special transfer casks.

The last category of casks is needed only in connection with dry-transfer facilities in reactors which do not have adequate crane capacity to handle large rail casks.

The key facilities that comprise the waste-transport system are:

- Reactor pools,

- Ery transfer facilities,

- Interim storage facilities,

- Independent Spent Fuel Storage Installation (ISFSI)

- Federal Interim Storage (FIS)

- Monitored Retrievable Storage (MRS)

- Railheads - off site,

- Barge siips

- Barge-rail transfer - off site, and the

- Repository.

When reactors are unioaded, the SNF is placed into storage pools to cool. when the SNF is ready to be shipped, it is wet-loaded in the pool into shipping casks and shipped off site. 
For long-term onsite storage, Independent Spent Fuel Storage Installations could be used in conjunction with transpcrtable storage casks. In this case, fuel would be first stored at the ISFSI in a storage cask and then shipped off site in the same cask. In another version, the fuel would be stored on site in a storage-only cask, then transferred to a rail cask and shipped off site.

Section $131(a)(3)$ of the NWPA also provides for Federal Interim Storage of not more than 1,900 MTU of SNF for civilian nuclear power reactors that "cannot reasonably provide adequate storage capacity at the sites of such reactors when needed to assure the continued, orderiy operation of such reactors." No FIS facilities are planned at this time.

Finally, an MRS facility is being considered as an option to provide safe and reliable management of SNF and HLW.

In the current study, reactors falling into certain kinds of categories (e.g., pressurized-water reactor (PWR), boiling-water reactor (BWR), truckserviced, etc.) are assumed to be lumped together into single nodes. These reactor nodes are characterized by the type of applicable available at-reactor waste handling and transport service category under consideration.

Because route information is basically a given in this study (see Section 3.3 ), the key waste transport system parameters can be reduced further to only three variables, i.e.,

- Vehicles,

- Casks, and

- Facilities.

The current study is confined to transportation options under existing site constraints and truck-rail options only. Thus the components of the waste transport system that need to be considered can be reduced to the following items:

- Vehicies

- Legal-weight trucks (LWT),

- Overweight trucks (OivT),

- Heavy-haul trucks (HHT), and

- Casks

- Railcars $(R)$.

- Legal-weight truck casks (LWTC),

- Overweight truck casks (OwTC),

- Regular rail casks (RC), and

- Hefty rail casks (HRC).

- Facilities

- Pools (P).

- Railheads (RH), and the

- Repository (REP).

With these 11 waste-system components, 7 transportation modal/intermodal options can be constructed for evaluation. These options are presented graphically in Figure 2-1, showing the point of origin, the vehicle and cask involved, and the final repository destination. Options (5) to (7) represent 
intermodal transport (IMT), because both trucks and rails are used for one shipment.

(1)

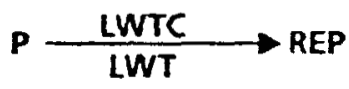

(2)

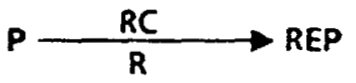

(3)

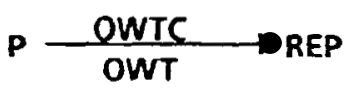

(4)

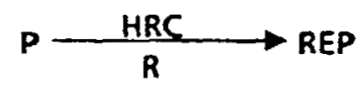

(5)

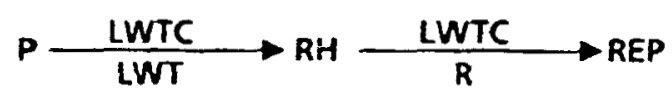

(6)

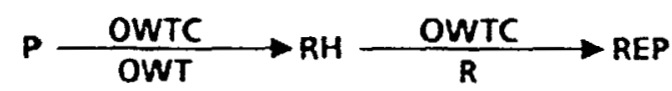

(7)

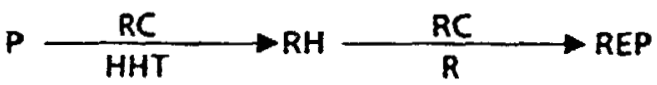

Figure 2-1. Transport Modes

\subsection{STUDY CASES}

The shipment options discussed in Section 2.1 have been organized into 7 cases. The cases are briefly described below.

\section{Case I}

All Truck (LWT) - This represents a somewhat artificial, but bounding case where it is postulated that all shipments from reactor pools to the repository are performed via LWTS.

\section{Case II}

Base case (LWT/R) - In the base case, reactors that can be serviced by rail are assumed to have their S:IF shipped in regular rail casks by rail: the remaining reactors are serviced by Lwis.

\section{Case III}

Overweight Trucks (LWT/OWT/R) - In this case, the truck-serviced reactors are divided into two groups: those that can handle OWTCS and those that can only handle LWTCS. The first group ships their SNF by OWT; the second group uses LWTs. The remaining casks are shipped via regular rail. 
Overweight/Hefty-Rail Casks (LWT/OWT/R/HRC) - In Case IV, the truckserviced reactors ship by OWTs or LWTs as in Case III. The rail-serviced reactors ship in HRCs wherever adequate crane capacity permits it. The remaining rail-serviced reactors ship in regular rail casks.

\section{Case V}

Overweight by Rail (LWT/OWT[R]/R/HRC) - This is the first IMT case. OWT[R] means OWT casks are shipped long distance by rail. In this case the casks are expected to be transpo ted by truck to the nearest railhead and transferred to railcars and then shipped by rail. The remainder of the shipments are treated as in Case IV.

\section{Case VI}

Overweight and Legal Weight by Rail (LWT[R]/OWT[R]/R/HRC) - In Case VI, an IMT case, it is assumed that all truck casks, LWTs and OW's, are trucked to the nearest $\mathrm{RH}$ and transferred to rail for shipment to the repository.

\section{Case VII}

Heavy-Haul Truck (HHT) - Case VII is divided into three subcases, all involving the use of HHTS. Among the reactors that are currentiy not serviced by rail, there are quite a few that do have the necessary crane capacity to handle regular and heavier rail casks but do not have direct rail access. In Case VII it is assumed that heavy-haul trucks can haul 100 -ton rail casks from these reactors to the nearest railhead for transfer to rail and shipment to the repository.

\section{Case VIIa}

(HHT[R]/OWT/LWT/R/HRC) - In the IMT Case VIIa, HHTs are used to haul 100ton raii casks to the nearest railhead for transfer from otherwise truckserviced nuclear plants. The remaining truck-serviced plants ship their SNF by LW'T and ONT.

\section{Case VIIb}

(HHT[R]/OWT[R]/LWT/R/HRC) - The IMT Case VIIb is like Case VIIa, except that the residual OWTCs are transshipped by rail from the nearest railhead.

\section{Case VIIC}

(HHT[R]/OWT[R]/LWT[R]/R/HRC) - The IMT Case VIIc is like Case VIIb, except that the LWTCS are also transshipped by rail from the nearest railhead.

\subsection{NUCLEAR PLANT DATA}

The study encompasses the entire U.S. reactor industry, including nuclear power plants as well as nuclear waste storage pools. Several U.S. nuclear 
capacity projections have been made for the intermediate term through 2000 , and the long term, through 2020. These projections, summarized in Reference (4), fall under the headings of a "Lower Reference," "Upper Reference, " and "Optimistic" case. One of the projections derived from these cases is the so-called "No New Orders" (NNO) Case, which consists of existing nuclear plants and nuclear power generating units in the construction pipeline, i.e., committed and likely to go on line, as of December 31, 1986. For this study, the No New Orders - 1986 (NNO-1986) case has been adopted as the starting point. This is appropriate because the site/facilities constraints important to and used in this study are "known." Pacific Northiest Laboratory (PNL) has listed and described reactors and storage pools covering this case in Reference (5), as well as lifetime SNF discharge data (in MTU).

For the study, it is further assumed that all nuclear plants can be lumped together into one or more reactor nodes. Distances for both truck and rail service from these nodes to the Nevada Yucca Mountain site have been taken from References (6) and (7). The reactor node-repository distances represent MIUweighted averages.

As part of the Reactor Data Analysis Progran's reactor-specific database task, PNL has provided shipping mode capability information for each reactor. The bases for this information are 1984, 1985, and $1986 \mathrm{RW}-859$ Nuclear Fuel Data Forms provided by the utilities. The reactor-specific cask handling capabilities are derived from these data and categorized as 25 tons or 40 tons if truck is the only form of transport. In case the data indicate that rail shipments are feasible, a distinction was made between 100-ton and 125-ton capability. For purposes of this study, the PNL shipping mode capability categorization has been adopted.

All of this information is summarized for 118 nuclear reactor plants and 7 nuclear waste storage pools in Table 2-1. Using the information in Table 2-1, total SNF discharge quantities in MTU and MTU-weighted average distances were calculated for specific c.tegories of reactors designated by mode of transport and by pool/cask handling capabilities. This information is summarized in Table 2-2.

As noted in rable 2-2, a total of 86,779 MTU shipments of SNF are encompassed in the NNO-1985 case. In terms of MTU shipped, the actual PWR/BWR split is $65 / 35$. This compares with a $60 / 40 \mathrm{split}$ assumed in the PNL ALARA Study (8). The actual life-cycle levelized rail/truck split is 56/44, which compares to the $70 / 30$ used in the 1937 PNL study. Inis comparison is noted to acknowledge the difference between current data and past assumptions.

The percentage cask handling capabilities for all facilities are as follows:

$$
\begin{aligned}
& 25-\text { ton handling } \\
& 40-\text { ton handling } \\
& 100-\text { ton handl ing } \\
& 125-\text { ton handling }
\end{aligned}
$$

$$
\begin{aligned}
& 100 \% \text { of all nuclear plants } \\
& 92.5 \% \text { of all nuclear plants } \\
& 58.7 \mathrm{z} \text { of all nuclear plants } \\
& 41.3 \% \text { of all nuclear plants. }
\end{aligned}
$$

The $25-t$ in truck cask can be handled at all reactor facilities. The 125-ton casks can be handled at 4.3 . of all nuclear plants. 
Table 2-1. Detalled Reactor/Storage Pool Data for the Transport Modal Option Study

\begin{tabular}{|c|c|c|c|c|c|c|c|}
\hline \multirow[b]{2}{*}{ Facility Name } & \multirow[b]{2}{*}{ State } & \multirow{2}{*}{$\begin{array}{l}\text { Type } \\
\text { of } \\
\text { Fuel }\end{array}$} & \multirow{2}{*}{$\begin{array}{c}\text { MTU } \\
\text { Oischarged } \\
\text { Thru Life }\end{array}$} & \multicolumn{2}{|c|}{$\begin{array}{c}\text { Distance to rucca Mtn. } \\
\text { site (mi) }\end{array}$} & \multirow{2}{*}{$\begin{array}{c}\text { Nominal } \\
\text { Handling } \\
\text { Capability } \\
\text { (tons) }\end{array}$} & \multirow{2}{*}{$\begin{array}{l}\text { Transport } \\
\text { Mode }\end{array}$} \\
\hline & & & & Highway & Ratl & & \\
\hline Arkansas Nuclear 1 & AR & PWR & 645.151 & 1744 & 2066.60 & 100 & $R$ \\
\hline Arkansas Nuclear 2 & $A R$ & PWR & 633.809 & 1744 & 2066.60 & 100 & $R$ \\
\hline Beaver Valley 1 & PA & PWR & 671.020 & 2347 & 2572.40 & 125 & $R$ \\
\hline Beaver Valley 2 & PA & PWR & 728.3810 & 2347 & 2572.40 & 125 & $R$ \\
\hline Bellefonte 1 & $A L$ & PWR & 804.769 & 2302 & 2602.40 & 125 & $R$ \\
\hline Big Rock 1 & MI & BWR & 73.226 & 2391 & 2481.00 & 25 & $T$ \\
\hline Braidwood 1 & IL & PWR & 822.735 & 1902 & 2060.80 & 125 & $R$ \\
\hline Braldwood 2 & IL & PWR & 813.006 & 1902 & 2060.80 & 125 & $R$ \\
\hline Browns Ferry 1 & $\mathrm{Al}$ & BWR & 1005.685 & 2279 & 2365.40 & 40 & $T^{\star}$ \\
\hline Browns Ferry 2 & A.L & BWR & 1040.852 & 2279 & 2365.40 & 40 & $T^{\star}$ \\
\hline Browns Ferry 3 & $\mathrm{AL}$ & BWR & 994.018 & 2279 & 2365.40 & 40 & $T^{\star}$ \\
\hline Brunswick 1 & $N C$ & BWR & 810.307 & 2836 & 3032.90 & 40 & $T$ \\
\hline Brunswick 1 Pool & NC & PWR & 71.342 & 2836 & 3032.90 & 40 & $T$ \\
\hline Brunswick 2 & NC & BWR & 774.827 & 2836 & 3032.90 & 40 & $T$ \\
\hline Brunswick 2 Pool & NC & PWR & 65.534 & 2836 & 3032.90 & 40 & $T$ \\
\hline 8yron 1 & IL & PWR & 815.121 & 1862 & 2095.30 & 125 & $R$ \\
\hline Byron 2 & IL & PWR & 810.468 & 1862 & 2095.30 & 125 & $R$ \\
\hline Callaway 1 & MO & PWR & 834.714 & 1807 & 1834.40 & 40 & $T$ \\
\hline Calvert Cliffs 1 & MD & PWR & 696.244 & 2714 & 2966.50 & 25 & $T$ \\
\hline Calvert Cliffs 2 & $M D$ & PWR & 712.914 & 2714 & 2966.50 & 25 & $T$ \\
\hline Catawba 1 & $S C$ & PWR & 835.106 & 2628 & 2819.00 & 125 & $R$ \\
\hline Catawba 2 & SC & PWR & 854.120 & 2628 & 2819.00 & 125 & $R$ \\
\hline Clinton 1 & IL & BWR & 783.595 & 1927 & 2077.30 & 100 & $R$ \\
\hline Comanche Peak 1 & $T X$ & PWR & 923.801 & 1747 & 1693.50 & 25 & $T$ \\
\hline Comanche Peak 2 & $T X$ & PWR & 913.499 & 1747 & 1693.50 & 25 & $T$ \\
\hline Cook 1 & MI & PWR & 876.887 & 1985 & 2077.90 & 100 & $R$ \\
\hline
\end{tabular}


Table 2-1. Detailed Reactor/Storage Pool Data for the Transport Modal Option Study (Cont.)

\begin{tabular}{|c|c|c|c|c|c|c|c|}
\hline \multirow[b]{2}{*}{ Facility Name } & \multirow{2}{*}{ State } & \multirow{2}{*}{$\begin{array}{l}\text { Type } \\
\text { of } \\
\text { fuel }\end{array}$} & \multirow{2}{*}{$\begin{array}{c}\text { MTU } \\
\text { Discharged } \\
\text { Thru Life }\end{array}$} & \multicolumn{2}{|c|}{$\begin{array}{c}\text { Distance to Yucca Mtn. } \\
\text { Site }(m l)\end{array}$} & \multirow{2}{*}{$\begin{array}{c}\text { Nominal } \\
\text { Handling } \\
\text { Capability } \\
\text { (tons) }\end{array}$} & \multirow{2}{*}{$\begin{array}{l}\text { Transport } \\
\text { Mode }\end{array}$} \\
\hline & & & & Highway & $\operatorname{Ra} 11$ & & \\
\hline Cook 2 & $M I$ & PWR & 796.613 & 1985 & 2077.90 & 100 & $\mathrm{R}$ \\
\hline Cooper Stn & NE & $B W R$ & 667.008 & 1516 & 1620.30 & 40 & $T$ \\
\hline Crystal River 3 & $\mathrm{FL}$ & PWR & 668.419 & 2837 & 3009.10 & 40 & $T$ \\
\hline Davis-Besse 1 & $\mathrm{OH}$ & PWR & 653.482 & 2186 & $\hat{\imath} 386.80$ & 125 & $R$ \\
\hline Diablo Canyon 1 & $C A$ & PWR & 663.559 & 546 & 686.50 & 40 & $T^{\star}$ \\
\hline Dlablo Canyon 2 & $C A$ & PWR & 660.293 & 546 & 686.50 & 40 & $T^{\star}$ \\
\hline Dresden 1 & IL & BWR & 69.525 & 1900 & 2003.20 & 40 & $\mathrm{~T}$ \\
\hline Dresden 2 & IL. & BWR & 748.421 & 1900 & 2003.20 & 100 & $R$ \\
\hline Dresden 3 & IL & BWR & 723.321 & 1900 & 2003.20 & 100 & $\mathbf{R}$ \\
\hline Duane Arnold & IA & BWR & 540.209 & 1721 & 1793.60 & 40 & $T$ \\
\hline Enrico Fermi 2 & MI & BWR & 1019.794 & 2179 & 2363.30 & 125 & $R$ \\
\hline Farley 1 & $A L$ & PWR & 778.500 & 2533 & 2956.10 & 125 & $R$ \\
\hline Farley 2 & AL & PWR & 727.170 & 2533 & 2956.10 & 125 & $R$ \\
\hline Fitzpatrick & NY & BWR & 789.705 & 2622 & 2805.10 & 125 & $R$ \\
\hline Fort Calhoun 1 & $N E$ & PWR & 410.754 & 1458 & 1530.60 & 40 & $T$ \\
\hline Ginna & NY & PWR & 443.867 & 2533 & 2751.50 & 25 & $T$ \\
\hline Grand Gulf 1 & MS & BWR & 1163.114 & 2080 & 2418.60 & 40 & $T$ \\
\hline Haddam Neck & $\mathrm{CT}$ & PWR & 535.127 & 2809 & 3104.30 & 40 & $T$ \\
\hline Harris 1 & NC & PiWR & 582.645 & 2732 & 2962.20 & 40 & $\mathrm{~T}$ \\
\hline Harris 1 Pool & $N C$ & BWP & 0.000 & $273 ?$ & 2962.20 & 40 & $\mathrm{~T}$ \\
\hline Hatch 1 & GA & BWR & 864.507 & 2619 & 2898.60 & 125 & $R$ \\
\hline Hatch 2 & GA & BWF & 844.062 & 2619 & 2898.60 & 125 & $\mathbf{R}$ \\
\hline Hope Creek 1 & $\mathrm{NJ}$ & BWR & 1066.180 & 2755 & 2946.50 & 40 & $T^{\star}$ \\
\hline Humboldt Bay & $C A$ & BWR & 28.942 & 1003 & 1157.80 & 40 & $\mathrm{~T}$ \\
\hline Indian Point 1 & NY & PNR. & 30.584 & 2728 & 3021.80 & 40 & $T$ \\
\hline
\end{tabular}


Table 2-1. Detailed Reactor/Storage Pool Data for the Transport Modal Option Study (Cont.)

\begin{tabular}{|c|c|c|c|c|c|c|c|}
\hline \multirow[b]{2}{*}{ Facility Name } & \multirow[b]{2}{*}{ State } & \multirow{2}{*}{$\begin{array}{l}\text { Type } \\
\text { of } \\
\text { Fuel }\end{array}$} & \multirow{2}{*}{$\begin{array}{c}\text { MTU } \\
\text { Discharged } \\
\text { Thru Life. }\end{array}$} & \multicolumn{2}{|c|}{$\begin{array}{c}\text { Distance to Yucca Mtn. } \\
\text { Site }(\mathrm{mi})\end{array}$} & \multirow{2}{*}{$\begin{array}{l}\text { Nominal } \\
\text { Handling } \\
\text { Capabflity } \\
\text { (tons) }\end{array}$} & \multirow{2}{*}{$\begin{array}{l}\text { Transport } \\
\text { Mode }\end{array}$} \\
\hline & & & & Highway & Rail & & \\
\hline Indian Point 2 & NY & PWR & 736.010 & 2728 & 3021.80 & 40 & $\mathrm{~T}$ \\
\hline Indian Point 3 & NY & PWR & 720.268 & 2728 & 3021.80 & 40 & $T$ \\
\hline Kewaunee & $W I$ & PWR & 515.215 & 2098 & 2213.50 & 40 & $T^{\star}$ \\
\hline Lacrosse & $W I$ & $B W R$ & 37.951 & 1944 & 2094.00 & 40 & $T$ \\
\hline LaSalle City l & IL & $B W R$ & 931.494 & 1875 & 2046.20 & 100 & $R$ \\
\hline LaSalle City 2 & IL & BWR & 961.581 & 1875 & 2046.20 & 100 & $R$ \\
\hline Limerick 1 & PA & BWR & 937.784 & 2755 & 2880.60 & 100 & $\mathrm{R}$ \\
\hline Maine Yankee & $M E$ & PWR & 767.259 & 3029 & 3242.90 & 100 & $R$ \\
\hline McGuire 1 & NC & PWR & 833.875 & 2597 & 2881.50 & 125 & $\mathrm{R}$ \\
\hline McGuire 2 & NC & PWR & 862.737 & 2597 & 2881.50 & 125 & $R$ \\
\hline Millstone 1 & $C T$ & BWR & 707.584 & 2813 & 3115.00 & 40 & $\mathrm{~T}$ \\
\hline Millistone 2 & $\mathrm{CT}$ & PWR & 650.149 & 2813 & 3115.00 & 100 & $\mathrm{R}$ \\
\hline Millstone 3 & $\mathrm{CT}$ & PWR & 883.642 & 2813 & 3115.00 & 125 & $R$ \\
\hline Monticello & MN & BWR & 376.738 & 1863 & 1932.70 & 40 & $T$ \\
\hline Morris-B Pool & IL & BWR & 432.020 & 1902 & 2001.20 & 125 & $R$ \\
\hline Morris-P Pool & IL & PWR & 132.062 & 1902 & 2001.20 & 125 & $R$ \\
\hline Nine Mile Point 1 & NY & BWR & 648.411 & 2623 & 2804.30 & 100 & $\mathrm{R}$ \\
\hline Nine Mile Point 2 & NY & $B W R$ & 882.165 & 2623 & 2804.30 & 125 & $R$ \\
\hline North Anna 1 & VA & PWR & 701.833 & 2741 & 2788.60 & 125 & $R$ \\
\hline North Anna 2 & VA & PWR & 700.328 & 2741 & 2788.60 & 125 & $R$ \\
\hline Oconee 1 & SC & PWR & 795.869 & 2535 & 2768.50 & 40 & $T \star$ \\
\hline Oconee 2 & SC & PWR & 671.682 & 2536 & 2768.50 & 40 & $T^{\star}$ \\
\hline Oconee 3 & SC & PWR & 761.666 & 2536 & 2768.50 & 40 & $T^{\star}$ \\
\hline Oyster Creek 1 & $\mathrm{NJ}$ & BWR & 644.192 & 2791 & 3030.40 & 40 & $T^{\star}$ \\
\hline Palisades & MI & PWR & 579.234 & 2014 & 2107.90 & 40 & $T$ \\
\hline
\end{tabular}


Table 2-1. Detalled Reactor/Storage Pool Data for the Transport Modal Option Study (Cont.)

\begin{tabular}{|c|c|c|c|c|c|c|c|}
\hline \multirow{2}{*}{ Facility Name } & \multirow[b]{2}{*}{ State } & \multirow{2}{*}{$\begin{array}{l}\text { Type } \\
\text { of } \\
\text { fuel }\end{array}$} & \multirow{2}{*}{$\begin{array}{c}\text { MTU } \\
\text { Discharged } \\
\text { Thru Life }\end{array}$} & \multicolumn{2}{|c|}{$\begin{array}{c}\text { Distance to Yucca Mtn. } \\
\text { Site (mi) }\end{array}$} & \multirow{2}{*}{$\begin{array}{l}\text { Nominal } \\
\text { Handling } \\
\text { Capabilitty } \\
\text { (tons) }\end{array}$} & \multirow{2}{*}{$\begin{array}{l}\text { Transport } \\
\text { Mode }\end{array}$} \\
\hline & & & & Highway & Rail & & \\
\hline Palo Verde 1 & $A Z$ & PWR & 807.628 & 607 & 695.50 & 125 & $R$ \\
\hline Palo Verde 2 & $A Z$ & PWR & 826.693 & 607 & 695.50 & 125 & $R$ \\
\hline Palo Verde 3 & $A Z$ & PWR & 823.657 & 607 & 695.50 & 125 & $R$ \\
\hline Peachbottom 2 & PA & BWR & 1010.950 & 2713 & 2859.60 & 40 & $T^{\star}$ \\
\hline Peachbottom 3 & PA & BWR & 975.533 & 2713 & 2859.60 & 40 & $T^{\star}$ \\
\hline Perry 1 & $\mathrm{OH}$ & BWR & 980.577 & 2290 & 2453.10 & 125 & $R$ \\
\hline Pilgrim 1 & MA & BWR & 645.724 & 2922 & 3203.00 & 25 & $T$ \\
\hline Point Beach 1 & WI & PWR & 446.767 & 2094 & 2145.50 & 40 & $T^{\star}$ \\
\hline Point Beach 2 & WI & PWR & 436.602 & 2094 & 2145.50 & 40 & $T^{\star}$ \\
\hline Prairie Island 1 & MN & PWR & 478.996 & 1858 & 1893.50 & 125 & $R$ \\
\hline Prairie Island 2 & $M N$ & PWR & 488.947 & 1858 & 1893.50 & 125 & $R$ \\
\hline Quad Cities 1 & IL & BWR & 790.904 & 1770 & 2012.00 & 100 & $R$ \\
\hline Quad Cities 2 & IL & BWR & 799.823 & 1770 & 2012.00 & 100 & $R$ \\
\hline Rancho Seco'1 & $C A$ & PWR & 617.827 & 680 & 821.00 & 40 & $T$ \\
\hline River Bend 1 & LA & BWR & 872.937 & 2134 & 2676.10 & 125 & $R$ \\
\hline Robinson 2 & SC & PWR & 503.986 & 2681 & 2865.00 & 40 & $T$ \\
\hline Salem 1 & NJ & PWR & 881.505 & 2755 & 2953.00 & 40 & $T^{\star}$ \\
\hline Salem 2 & NJ & PWR & 887.176 & 2755 & 2953.00 & 40 & $T^{\star}$ \\
\hline San Onofre 1 & $C A$ & PWR & 271.284 & 415 & 455.00 & 25 & $T$ \\
\hline San Onofre 2 & $\mathrm{CA}$ & PWR & 802.402 & 415 & 455.00 & 125 & $\mathbf{R}$ \\
\hline San Onofre 3 & $C A$ & PWR & 815.707 & 415 & 455.00 & 125 & $\mathrm{R}$ \\
\hline Sequoyah 1 & $\mathrm{TN}$ & PWR & 821.451 & 2318 & 2537.00 & 40 & $T^{\star}$ \\
\hline Sequoyah 2 & TN & PWR & 825.486 & 2318 & 2537.00 & 40 & $T^{\star}$ \\
\hline South Texas 1 & $i x$ & PWR & 979.757 & 1862 & 2613.20 & 125 & $R$ \\
\hline South Texas 2 & $T X$ & PWR & 974.680 & 1862 & 2613.20 & 125 & $R$ \\
\hline
\end{tabular}


Table 2-1. Detafled Reactor/Storage Pool Data for the Transport Modal Option Study (Cont.)

\begin{tabular}{|c|c|c|c|c|c|c|c|}
\hline \multirow[b]{2}{*}{ Facility Name } & \multirow[b]{2}{*}{ State } & \multirow{2}{*}{$\begin{array}{c}\text { Type } \\
\text { of } \\
\text { Fuel }\end{array}$} & \multirow{2}{*}{$\begin{array}{c}\text { MTU } \\
\text { Discharged } \\
\text { Thru Life }\end{array}$} & \multicolumn{2}{|c|}{$\begin{array}{c}\text { Distance to Yucca Mtn. } \\
\text { Site (mi) }\end{array}$} & \multirow{2}{*}{$\begin{array}{c}\text { Nominal } \\
\text { Handling } \\
\text { Capab } 111 \text { ty } \\
\text { (tons) }\end{array}$} & \multirow{2}{*}{$\begin{array}{l}\text { Transport } \\
\text { Mode }\end{array}$} \\
\hline & & & & Highway & $\operatorname{Rat1}$ & & \\
\hline St. Lucte 1 & FL & PWR & 731.755 & 2957 & 3116.10 & 25 & $T$ \\
\hline St. Lucie 2 & $\mathrm{FL}$ & PWR & 703.902 & 2957 & 3116.10 & 40 & $T^{\star}$ \\
\hline Summer 1 & SC & PWR & 768.960 & 2600 & 2779.00 & 125 & $R$ \\
\hline Surry 1 & VA & PWR & 640.287 & 2843 & 2890.50 & 40 & $T^{\star}$ \\
\hline Surry 2 & VA & PWR & 597.208 & 2843 & 2890.50 & 40 & $T^{\star}$ \\
\hline Susquehanna 1 & $P A$ & BWR & 987.985 & 2565 & 2879.60 & 125 & $R$ \\
\hline Susquehanna 2 & PA & BWR & 995.304 & 2565 & 2879.60 & 125 & $R$ \\
\hline Three Mile Island 1 & $P A$ & PWR & 691.850 & 2666 & 2814.60 & 100 & $R$ \\
\hline Trojan & OR & PWR & 792.295 & 1328 & 1475.70 & 125 & $R$ \\
\hline Turkey Point 3 & $F L$ & PWR & 588.917 & 3103 & 3340.10 & 25 & $T$ \\
\hline Turkey Point 4 & $F L$ & PWR & 559.131 & 3103 & 3340.10 & 25 & $\mathrm{~T}$ \\
\hline Vermont Yankee & VT & BWR & 652.385 & 2848 & 3088.90 & 100 & $R$ \\
\hline Vogtle 1 & $G A$ & PWR & 912.559 & 2606 & 2933.70 & 125 & $R$ \\
\hline Vogtle 2 & GA & PWR & 921.370 & 2606 & 2933.70 & 125 & $\mathrm{R}$ \\
\hline Wash Nuclear 2 & WA & BWR & 984.099 & 1166 & 1309.70 & 40 & $T^{\star}$ \\
\hline Waterford 3 & LA & PWR & 858.317 & 2200 & 2610.70 & 125 & $R$ \\
\hline Watts Bar 1 & TN & PWR & 797.298 & 2368 & 2502.00 & 40 & $T^{\star}$ \\
\hline Watts Bar 2 & TN & PWR & 785.767 & 2368 & 2502.00 & 40 & $T^{\star}$ \\
\hline West Valley-B Pool & NY & BWR & 11.497 & 2452 & 2651.90 & 100 & $R$ \\
\hline West Valley-P Pool & NY & PWR & 15.287 & 2452 & 2651.90 & 100 & $R$ \\
\hline Wolf Creek & KS & PWR & 851.220 & 1669 & 1765.60 & 125 & $R$ \\
\hline Yankee-Rowe 1 & MA & PWR & 165.707 & 2885 & 2967.50 & 40 & $T$ \\
\hline Zion 1 & IL & PWR & 821.997 & 1955 & 2023.10 & 125 & $R$ \\
\hline 2 ion 2 & IL & PWR & 817.841 & 1955 & 2023.10 & 125 & $R$ \\
\hline
\end{tabular}

*Truck-serviced reactor plants that have potential crane capacity to handle rail casks > 100 tons. 
Table 2-2. Aggregated Reactor/Storage Pool Data for the Transport Modal Options Study

\begin{tabular}{|c|c|c|c|c|c|c|}
\hline $\begin{array}{c}\text { Service } \\
\text { Redctor/Pool class }\end{array}$ & $\begin{array}{c}\text { Number } \\
\text { of } \\
\text { Facilities }\end{array}$ & $\begin{array}{c}\text { SNF } \\
\text { PWR/BWR }\end{array}$ & $\begin{array}{l}\text { MIU Discharges } \\
\text { Through Life }\end{array}$ & $\begin{array}{l}\text { Distand } \\
\text { Mounte } \\
\text { Híghway }\end{array}$ & $\frac{\text { Aes) }}{\text { Rait }}$ & $\begin{array}{c}\text { Initid] } \\
\text { Iransportation } \\
\text { Mode } \\
I / R\end{array}$ \\
\hline \multirow[t]{2}{*}{ LWT, 25 tons } & $\begin{array}{l}9 \\
2\end{array}$ & $\begin{array}{l}\text { PWR } \\
\text { BUR }\end{array}$ & $\begin{array}{r}5841 \\
719\end{array}$ & $\begin{array}{l}2396 \\
2868\end{array}$ & $\begin{array}{l}2525 \\
3129\end{array}$ & $\begin{array}{l}T \\
T\end{array}$ \\
\hline & $\pi$ & PWR/BWK & 6560 & 2448 & 2592 & $T$ \\
\hline \multirow[t]{2}{*}{ Ow1. 40 tons } & $\begin{array}{l}31 \\
19\end{array}$ & $\begin{array}{l}\text { PWR } \\
\text { BWR }\end{array}$ & $\begin{array}{l}18414 \\
12898\end{array}$ & $\begin{array}{l}2297 \\
2325\end{array}$ & $\begin{array}{l}2464 \\
2490\end{array}$ & $\begin{array}{l}\mathrm{T} \\
\mathrm{T}\end{array}$ \\
\hline & 50 & PWR/BWR & $3 \longdiv { 3 1 2 }$ & 2309 & 2475 & $T$ \\
\hline \multirow[t]{2}{*}{ Irucks } & $\begin{array}{l}40 \\
21 \\
\end{array}$ & $\begin{array}{l}\text { PWR } \\
\text { BWR }\end{array}$ & $\begin{array}{l}24255 \\
13617\end{array}$ & $\begin{array}{l}2321 \\
2379\end{array}$ & & $\begin{array}{r}T \\
T\end{array}$ \\
\hline & 61 & PWR/BWR & 37872 & 2333 & & $T$ \\
\hline$R, 100$ tons & $\frac{11}{19}$ & $\begin{array}{c}\text { PWR } \\
\text { BWR } \\
\text { PWR/BWR }\end{array}$ & $\begin{array}{r}5077 \\
7989 \\
13066\end{array}$ & & $\begin{array}{l}2486 \\
2280 \\
2360\end{array}$ & $\begin{array}{l}R \\
R \\
R\end{array}$ \\
\hline R, HRC, 125 tons & $\begin{array}{r}35 \\
10 \\
45\end{array}$ & $\begin{array}{c}\text { PWR } \\
\text { BWR } \\
\text { PWR/BWR }\end{array}$ & $\begin{array}{r}27171 \\
8669 \\
35840\end{array}$ & & $\begin{array}{l}2215 \\
2696 \\
2331\end{array}$ & $\begin{array}{l}\mathbf{R} \\
\mathbf{R} \\
\mathbf{R}\end{array}$ \\
\hline \multirow[t]{2}{*}{ Rail } & $\begin{array}{r}43 \\
21 \\
-\end{array}$ & $\begin{array}{l}\text { PWR } \\
\text { BWR }\end{array}$ & $\begin{array}{l}32248 \\
16658\end{array}$ & & $\begin{array}{l}2258 \\
2473\end{array}$ & $\begin{array}{l}\mathbf{R} \\
\mathbf{R}\end{array}$ \\
\hline & 64 & PWR/BWR & 48906 & & 2339 & $R$ \\
\hline \multirow[t]{2}{*}{$\begin{array}{l}\text { All Facilities } \\
\text { (All Truck Case) }\end{array}$} & $\begin{array}{r}83 \\
42\end{array}$ & $\begin{array}{l}\text { PWR } \\
\text { BWR }\end{array}$ & $\begin{array}{l}56504 \\
30275\end{array}$ & $\begin{array}{l}2155 \\
2312\end{array}$ & & $\begin{array}{l}T \\
T\end{array}$ \\
\hline & 125 & PWK/BWR & 86779 & 2210 & & $T$ \\
\hline
\end{tabular}




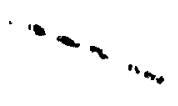

Table 2-2 (Cont.) Aggregated Reactor/Storage Pool Datia for the Transport Modal Options Study

\begin{tabular}{|c|c|c|c|c|c|c|}
\hline \multirow[t]{3}{*}{$\begin{array}{c}\text { Service } \\
\text { Reactor/Pool class }\end{array}$} & \multirow{3}{*}{$\begin{array}{c}\begin{array}{c}\text { Number } \\
\text { of } \\
\text { Facilities }\end{array} \\
17 \\
8 \\
-25\end{array}$} & \multirow{3}{*}{$\begin{array}{c}\begin{array}{c}\text { SNF } \\
\text { PWR/BWR }\end{array} \\
\text { PWR } \\
\text { BWR } \\
\text { PWR/BWR }\end{array}$} & \multirow{3}{*}{$\begin{array}{c}\text { MTU Discharges } \\
\text { Ihrough Life } \\
\begin{array}{r}1189 ? \\
1722 \\
19614\end{array}\end{array}$} & \multicolumn{2}{|c|}{$\begin{array}{l}\text { Distance to Yucca } \\
\text { Mountain (Miles) } \\
\text { Highway }\end{array}$} & \multirow{2}{*}{$\begin{array}{c}\begin{array}{c}\text { Initial } \\
\text { Transportation } \\
\text { Mode } \\
T / R\end{array} \\
T \\
T\end{array}$} \\
\hline & & & & & $\begin{array}{l}2460 \\
2494\end{array}$ & \\
\hline & & & & & 2473 & $T$ \\
\hline \multirow[t]{2}{*}{ Remaining OWT } & $\begin{array}{l}14 \\
11\end{array}$ & $\begin{array}{l}\text { PWR } \\
\text { BWR }\end{array}$ & $\begin{array}{l}6522 \\
5176 \\
\end{array}$ & $\begin{array}{l}2294 \\
2276\end{array}$ & $\begin{array}{l}2473 \\
2483\end{array}$ & $\begin{array}{l}\mathrm{T} \\
\mathrm{T}\end{array}$ \\
\hline & 25 & PWR/BWR & $\sqrt{1698}$ & 2286 & 2478 & $\mathbf{T}$ \\
\hline \multirow[t]{2}{*}{ Remaining LWT } & $\begin{array}{l}9 \\
2 \\
\end{array}$ & $\begin{array}{l}\text { PWR } \\
\text { BWR }\end{array}$ & $\begin{array}{r}5841 \\
119 \\
\end{array}$ & $\begin{array}{l}2396 \\
2868\end{array}$ & $\begin{array}{l}2525 \\
3129\end{array}$ & $\begin{array}{l}T \\
T\end{array}$ \\
\hline & $\pi$ & PWR/BWR & 6560 & 2448 & 2592 & $T$ \\
\hline
\end{tabular}




\subsection{STUOY BASES AND ASSUMPTIONS}

\subsection{CASK DATA}

A set of reference cask designs have been adopted for this study. The cask specifications in terms of loaded weight and PWR/BWR spent fuel assembly capacity are summarized in Table 3-1.

Table 3-1. Reference Cask Designs for the Transport Modal Option Study

\begin{tabular}{lcc}
\hline Type of Cask & Weight (tons) & Capacity \\
\hline LWTC & 28 & $3 / 7$ \\
OWTC & 40 & $5 / 12$ \\
Rail Cask, RC & 100 & $21 / 48$ \\
Hefty Rail Cask, HRC & 125 & $24 / 60$ \\
\hline
\end{tabular}

\subsection{COST DATA AND METHODS}

The LCC analyses for this study are being carried cut via the computer model CASKCOM (9). Using CASKCOM, component costs are calculated and then summed to obtain a total LCC. A description of the model assumptions and the model logic is given in the appendix. Key cost data used in estimating LCCs are summarized in Table 3-2.

Table 3-2. Key Cost Data

\begin{tabular}{|c|c|c|c|c|}
\hline & LWTC & OWTC & $R C$ & HRC \\
\hline Cask Unit Costs (Mi11 1987 \$) & .80 & 1.0 & 2.0 & 2.5 \\
\hline $\begin{array}{l}\text { Development, Certification Costs } \\
\quad(M i 111987 \mathrm{~s})\end{array}$ & 6.0 & 7.0 & 3.0 & 9.0 \\
\hline $\begin{array}{l}\text { Annual Unit Maintenance Costs } \\
\text { (Mill } 1987 \$)\end{array}$ & .075 & .085 & .125 & .150 \\
\hline $\begin{array}{l}\text { Crew Wages at Reactor and Repository } \\
(1987 \mathrm{~S} / \mathrm{hr})\end{array}$ & 63.0 & 63.0 & 63.0 & 63.0 \\
\hline
\end{tabular}


In current practice, only single-mode transport of SNF has been seriously considered. However, because many nuclear power plants are not directly accessible by rail but have rail cask handling capability, truck/rail IMT systems are now receiving more serious consideration. Cases $V$ to VII of this study specifically deal with IMT.

The cost calculations for IMT are done exogenously to CASKCOM. Two situations have been considered; so-called lift-off/lift-on (LO/LO) operations which pertain specifically to cases $V$ and $V I$; and roll-off/roll-on (RO/RO) operations which pertain to the HHT/rail transfers in Case VII.

For the LO/LO operations, it is assumed that truck casks (either LWTCs or OWTCS) are hauled by truck to the nearest railhead and ther transferred and shipped. The cask shipments would be part of a reactor annual shipping campaign, involving about 35 MTU. The number of casks per campaign would be about 25 LWTC loads or 15 OWTC loads, respectively.

It was further assumed that utility crew schedules and riggers' schedules are adequately optimized so that neither organization has to unreasonably wait for the other. Truck casks are shipped, one per truck, and loaded two per railcar.

For the LWTC cask, the cost of the IMT is estimated to be $\sim 3.5 \$ / \mathrm{kg}$. For the OWTC cask, the transfer costs are estimated to be $\sim 2.2 \$ / \mathrm{kg}$. The details of these cost estimates are shown in Table 3-3.

For the RO/RO operations, 100-ton rail casks are hauled by a HHT to the nearest railhead and then transferred to a railcar. There would be about 3 railcars per campaign. The casks would be shipped nne per HHT and loaded one per railcar. The cost of the RO/RO operation was estimated to be $\sim 2.4 \$ / \mathrm{kg}$. Details of this estimate are shown in Table 3-4.

\subsection{DOSE DATA AND METHODS}

To estimate collective occupational and public doses, use has been made of the methodology and data developed in the recent PNL ALARA study, published in November 1987 (8). In this method, doses in person-mrem are calculated for direct exposure from "cask work" as well as for exposure from "general area work" (background radiation). Different dose estimates are made for truck and rail casks and for PWR and BWR casks. Tre public incurs doses only from actual transit operations off site. Public doses resulting from reactor plant and repository operations are assumed to be negligible. The PNL in-transit doses are based on RADTRAN III (10) calculations for typical truck distances (i.e., 1,780 miles) and rail distances (i.e., 1,910 miles). In the calculations for this report, a simple distance correction nas been made for the in-transit doses, i.e., the in-transit doses have been assumed to be simply proportional to distance.

The dose data derived from the PNL report are summarized in Table 3-5. OWT shipments are expected to proceed differently than regular LWT shipments. There are more restrictions on OWT than on LWT. Increased stop-times compared to LWT shipments occur. The net result is an increase in overall public and occupational in-transit doses. The numbers in parentheses in Table 3-5 refer to ONT. 
Table 3-3. Estimated Costs for Truck/Rail

LO/LO Cask Transfer

\section{LWTC}

Crew size 5

Total working time, days (1 day/cask) 25

Total man-hours worked (5 men) (25 days) (10 hrs/day) 1,250

$\cos (s(\xi K)$

Labor costs (1250) (63 $\mathrm{s} / \mathrm{hr})$

$\sim 79$

Per diem (5) (25 days) (60 $\$ /$ day)

$\sim 8$

Mobilization and Return

(2 trips) [(5 men) (12 hrs/trip) $(63 \mathrm{~s} / \mathrm{hr})+(5)(60)]$

$\sim 8$

Crane rental (27 days) (1000 \$/day)

$\sim 27$

Truck rental (27 days) (75 \$/day)

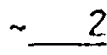

OWIC

$$
\$ / \mathrm{kg} \sim 124 / 35 \sim \underline{\underline{3.5}}
$$

Total

$\sim 124$

Crew size

Total working time, days (1 day/cask)

Total man-hours (5 men) (15 days) (10 hrs/day)

750

Costs $(5 K$

Labor costs $(750)(63 \mathrm{~s} / \mathrm{hr})$

$\sim 47$

Per diem (5) (15 days) (60 \$/day)

$\sim 5$

Mobilization and Return

(2 trips) [(5 men) (12 hrs/trip) $(63 \$ / \mathrm{hr})+(5)(60)] \sim 8$

Crane rental (17 days) (1000 \$/day)

$\sim 17$

Truck rental (17 days) (75 5/day)

$\sim$

$$
5 / \mathrm{kg} \sim 78 / 35 \sim 2.2
$$

Total

$-78$ 
Table 3-4. Estimated costs for HHT/Rail

RO/RO Cask Transfer

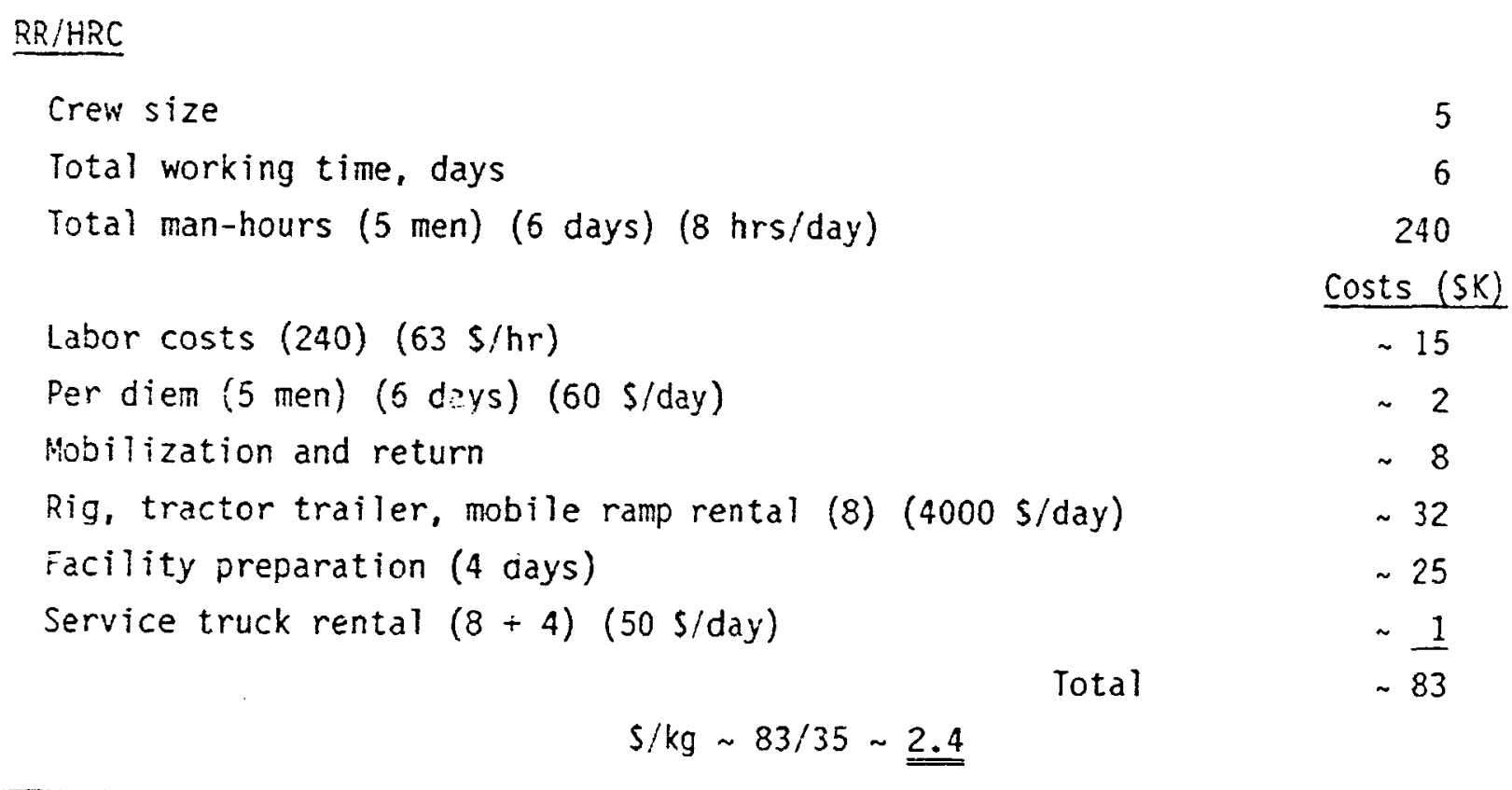

Table 3-5. Population Dose Summaries for Spent Fuel Transportation System

PWR

BWR

Iruck Doses (pers-mrem/cask)

Reactor Plant

Transit - Worker

Transit - Public*

Repository

Rail Doses (pers-mrem/cask)

\section{Reactor Plant}

Transit - Worker

Transit - Public

Repository

$261+5 P_{T}$
$206(218)$
$457(527)$
$276+.175 P_{T}$

$268+5 \mathrm{~B}_{T}$

$206(218)$

$457(527)$

$276+.175 \mathrm{~B}_{\mathrm{T}}$

where $P_{T}=$ number of PWR spent fuel assemblies (SFAS) per truck cask,

$B_{T}=$ number of BWR SFAs per truck cask,

$P_{R}=$ number of PWR SFAS per rail cask, and

$B_{R}=$ number of BWR SFAs per rail cask.

* Numbers in parentheses refer to ONT. 

casks.

The dose rates for the $H R C$ are assumed to be the same as for regular rail

For the IMT cases, a special set of dose data had to be developed. As in the cost section, separate dose estimates were developed for the $10 / 10$ and the RO/RO transfer operations.

For the LO/LO operations, a flow sheet for the truck to rail IMT is shown in Figure 3-1. It consists of 9 basic steps, starting with trucks hauling LWT/OWT casks to the nearest railhead (from nearby, up to 40 miles away) and ending with the return of the trucks to the reactor.

The casks are expected to be mounted on skids at the reactor, with the personnel barriers in place. Lifting of casks from the trucks to the railcars would be accomplished via 50-ton mobile cranes.

For each of the steps, dose rates and number of workers doing actual "cask work" or being in the "general area" have been estimated. Elapsed time for each operation was also estimated and used to calculate total collective dose in person-mrem for the transfer operations. This is summarized in Table 3-6.

Table 3-6. Collective Dose Data - Truck/Rail LO/LO Transfer

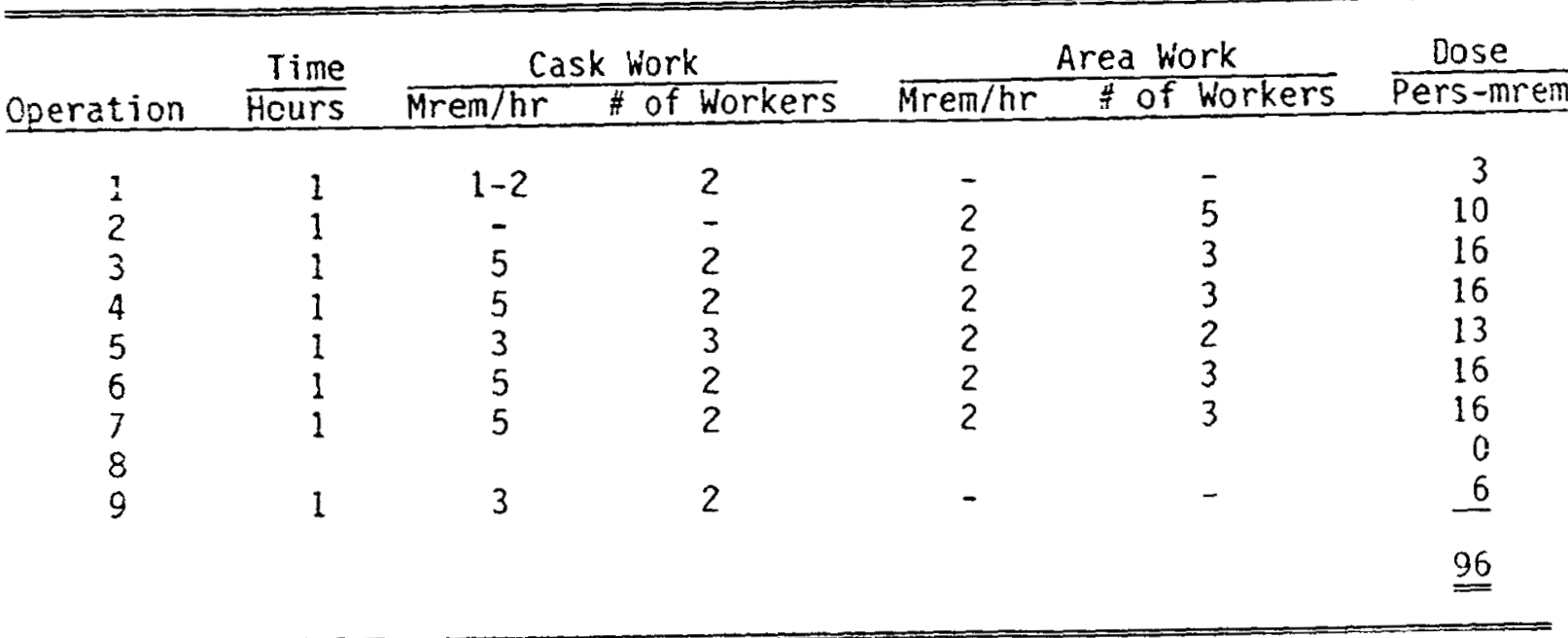

As shown in Table 3-6, the total dose for the truck/rail transfer operation is -96 person-mrem per cask.

Because the RO/RO operations are somewhat complex, some explanations and illustrations are in order. To start the RO/RO operations, a suitable heavyhaul transporter and accessory equipment are needed. These items are illustrated in Figure 3-2 [based on Reference (11)]. The cask mounted on a skid is sitting on a cart outside of the reactor bay. When the HHT is ready on site, the front and rear goosenecks are engaged to the equipment skid and the skid is raised to operating height. This is shown on Figure 3-3a. The cart is removed and the HHT hauls the cask/skid assembly to the railhead. At the railhead, the railcar is positioned adjacent to the loading ramp, the temporary ramps are installed, and the HHT is driven up the ramp (see figure 3-3b). 
1

Haul cask by LWT/OWT from reactor to railhead

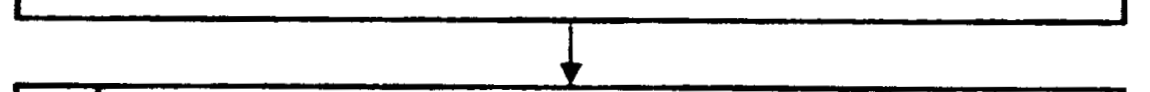

2 Receive LWT/OWT cask-vehicle at railhead

Position alongside railcar and crane

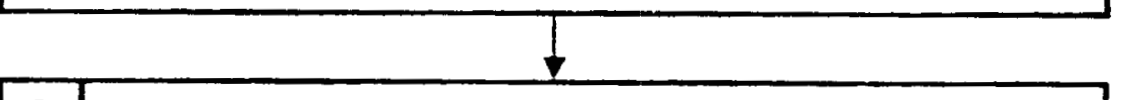

3

Remove skid tie-downs from trailer, ai four points

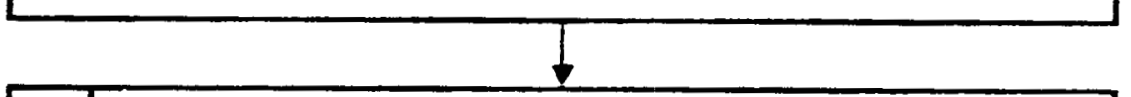

4

Attach lifting yoke to skid

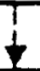

5

Lift cask-skid assembly from truck and position on railcar

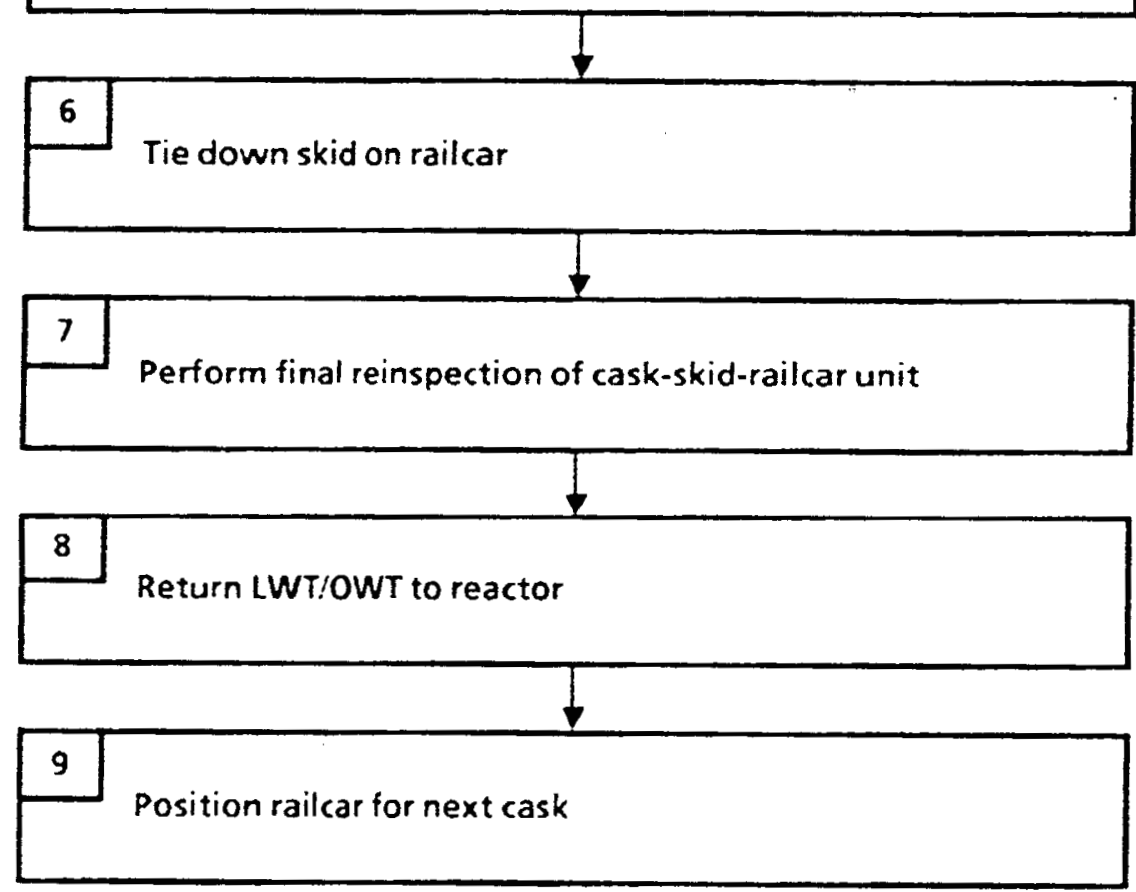

Figure 3-1. Truck-to-Rail Cask Transfer Operations (LOLO) 

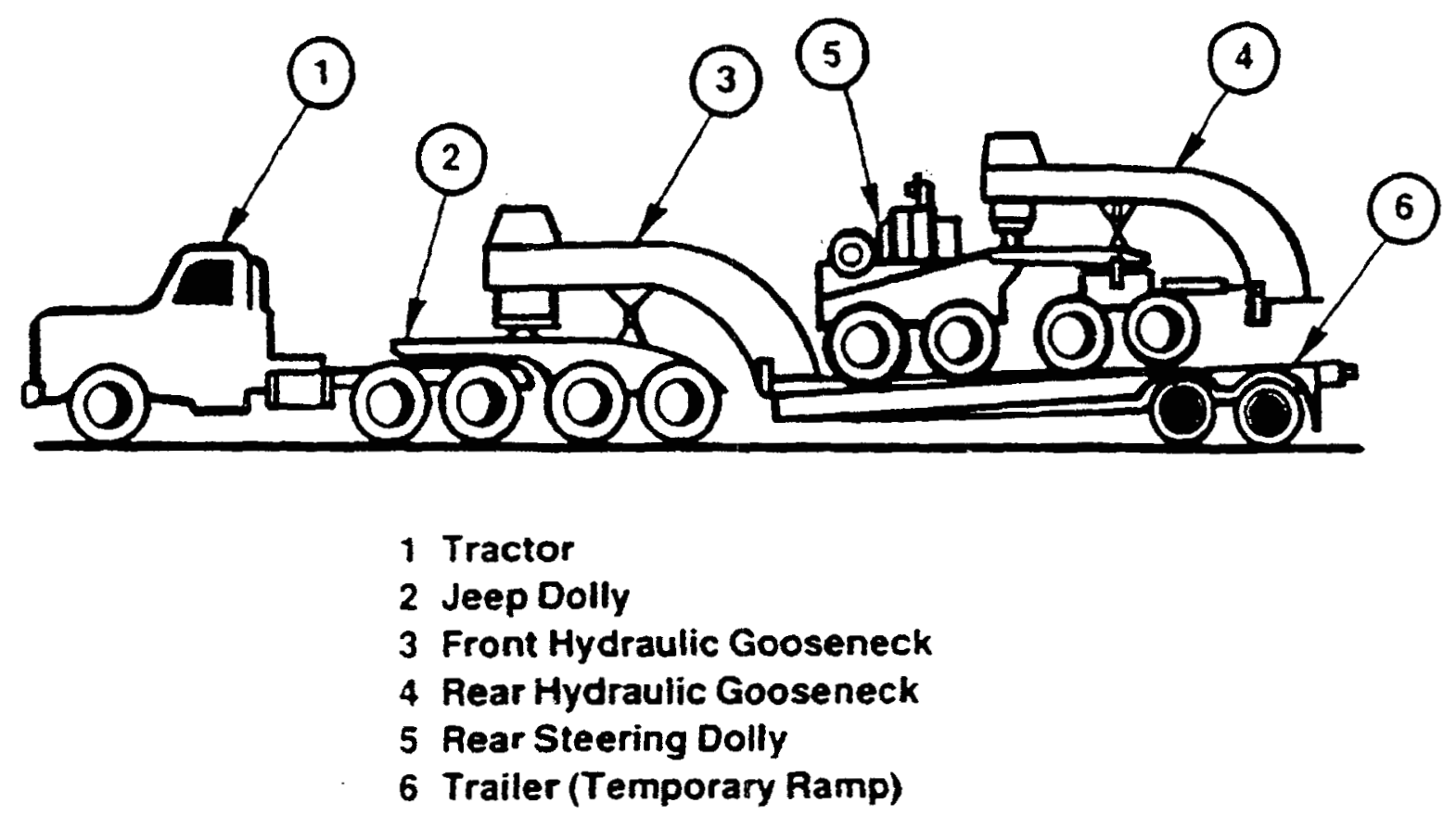

Figure 3-2. HHT Traisporter and Auxiliary Equipment [Based on Reference (11)]

To complete the operation, the cask/skid assembly is lowered onto the railcar (Figure 3-3c). The front and rear goosenecks are then disengaged, the cask/skid assembly is tied down, and after final inspections, the railcar can be moved to make room for the next cask operation. Finally, the tractor and goosenecks are returned to the reactor site.

For the RO/RO operation (Case VII) a suitable flow sheet was developed (Figure 3-4). It is assumed that at the start of the operation, the cask with personnel barriers in place has been mounted on a skid and is sitting on a transfer cart in the reactor loading bay.

The transfer operation has been subdivided into 12 steps, starting with the pull-out of the cart into a clear area outside of the reactor bay. In the last step of the operation, the tractor and goosenecks are returned to the reactor site.

For each of the 12 steps, dose rates, number of workers doing actual "cask work" or being in the "general area" have been estimated and used to calculate collective dose in person-mrem for the transloading operation. The dose data are summarized in Table 3-7.

The total dose for this operation is estimated to be $~ 94.5$ person-mrem. Pub1 ic dose is considered to be negligible. A similar analysis vias made in 1978 for a rail/barge transfer of a rail cask (12). For this case, the dose per cask was estimated to be -120 person-mrem. 


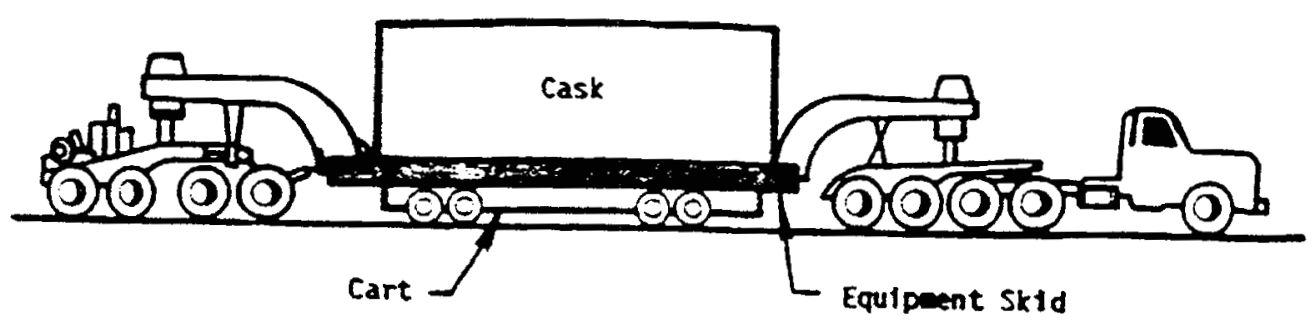

(a) HHT and Cask/Skid Assembly Ready for Transport

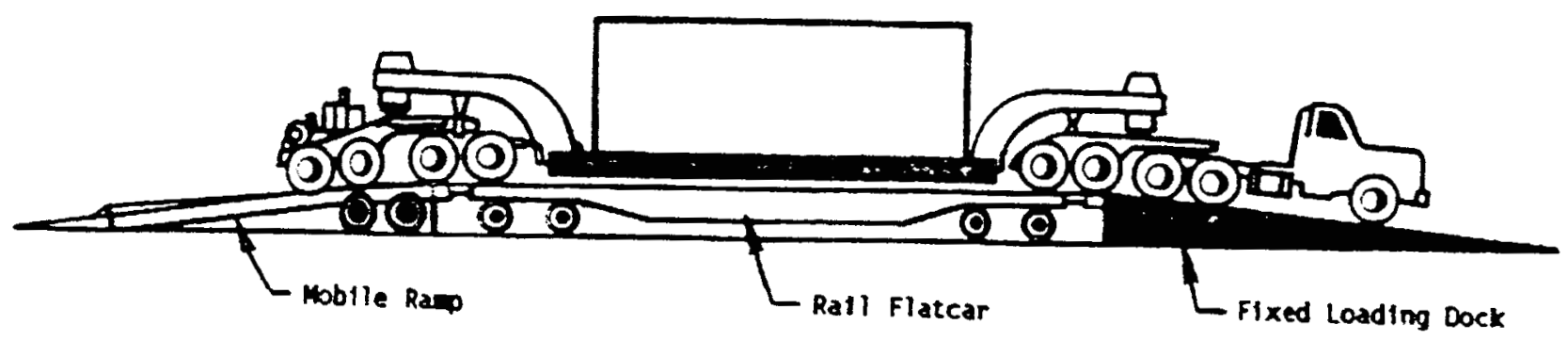

(b) Equipeent Skid Ready to be Lowered Onto the Railcar

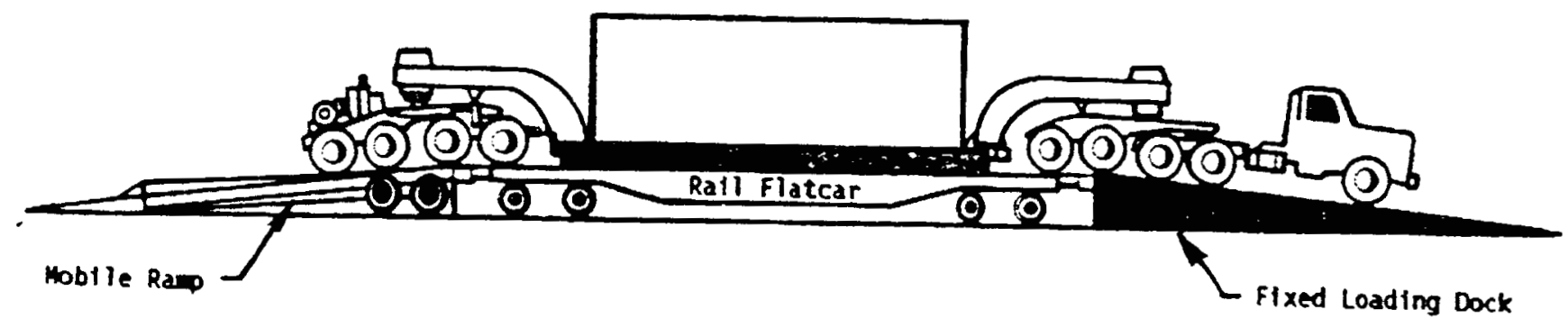

(c) Equipment skid in Place on Rallcar: Goosenecks Ready to Be Olsengaged at Iransfer Site

Figure 3-3. RO/RO Operations [Based on Reference (11)] 
1

Pull cart * out into a clear area outside the reactor bay

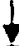

2 Engage front and rear goosenecks to skid and raise to operating height

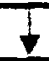

Clear cask for offsite transport

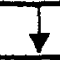

4

Haul caskiskid assembly to railhead

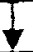

5 Position railcar adjacent to loading ramp and install temporary ramps

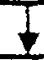

6 Drive tractor/cask/skid assembly up ramp onto railcar and position skid

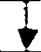

7 Lower cask/skid assembly onto railcar and disengage front and rear goosenecks

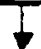

8

Tie down cask/skid assembly to railcar

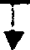

9 Connect loaded cask'skid/railcar assembly and train

$+$

10 Perform final reinspection of caskiskidirailcar unit

1

11

Return tractor and goosenecks to reactor site

1

12 Position railcar and temporary ramp for next cask

- Most reactor loading bays are not long enough to accommodate the HHT.

Figure 3-4. HHT-to-Rail Cask Transfer Operations (RO/RO) 
Table 3-7. Collective Dose Data - HHT/Rail RO/RO Transfer

\begin{tabular}{|c|c|c|c|c|c|c|c|}
\hline \multirow[b]{2}{*}{ Operation } & \multirow{2}{*}{$\begin{array}{c}\text { Time } \\
\text { (Hours) }\end{array}$} & \multicolumn{2}{|c|}{ Cask Work } & \multicolumn{3}{|c|}{ Area Work } & \multirow{2}{*}{$\frac{\text { Dose }}{\text { Pers-mrem }}$} \\
\hline & & Mrem/hr & $\#$ of Workers & Mrem/hr & $\ddot{\#}$ of & Workers & \\
\hline 1 & $1 / 2$ & - & - & 1 & & 1 & .5 \\
\hline 2 & 1 & 5 & 2 & 1 & & 1 & 11 \\
\hline 3 & 1 & - & - & 1 & & 4 & 4 \\
\hline 4 & 2 & 1 & 3 & - & & - & 6 \\
\hline 5 & 1 & - & - & 2 & & 5 & 10 \\
\hline 6 & 1 & 5 & 2 & 1 & & 3 & 13 \\
\hline 7 & 1 & 5 & 2 & 1 & & 3 & 13 \\
\hline 8 & 1 & 5 & 3 & 1 & & 2 & 17 \\
\hline 9 & 1 & - & - & 1 & . & 4 & 4 \\
\hline 10 & 1 & 5 & 2 & 2 & & 3 & 16 \\
\hline 11 & 1 & - & - & 0 & & 3 & 0 \\
\hline 12 & 1 & - & - & 0 & & 4 & 0 \\
\hline & & & & & & & 94.5 \\
\hline
\end{tabular}




\subsection{LIFE-CYCLE COST (LCC) ANALYSES}

As described in Chapter 3, life-cycle cost (LCC) analyses were performed using the CASKCOM computer program. Results of the analyses for each of the seven cases and three subcases of this program are presented in Tables 4-1 to 4-9. All estimates are total life-cycle costs in million of 1987 dollars. Detailed input data and cost formulas are compiled in the appendix.

A first examination of the results shows that, for single-mnde transport (Cases I to IV), the costs drop as the casks get larger and as fewer trips are needed to transport a given amount of fuel from the nuclear puwer plants to the repository. This trend is not completely followed as use is made of the hefty rail cask. In this case, the cost figures actually increase from $\sim 14 \$ / \mathrm{kg}$ (Case III) to $\sim 16 \mathrm{~s} / \mathrm{kg}$ for Case IV. At first, this seems counter-intuitive, because the HRC has a capacity of 24/60, which is larger than the capacity of $21 / 48$ for the regular rail cask. Thus, fewer trips and lower costs are expected. However, unlike the situation with regular rail casks, hefty rail casks are assumed to use dedicated train transportation for the entire round trip. Regular rail casks would travel by dedicated train only in the loaded condition.

When returning the empty casks, regular casks would be returned via regular trains at lower average speeds. The dedicated train charge is taken as $\$ 48 /$ train-mile and this adds considerably to the hauling cost. In fact, for Case IV, this cost differential has been calculated to be $\sim \$ 109$ million. If the hefty rail casks were assuried to return by regular train, the cost for case IV would only be $\$ 1,350$ minus $\$ 109 \sim \$ 1,251$ million, which is just slightly below the quoted Case III cost of $\$ 1,254$ million (Table 4-3).

It is interesting to compare the 1987 dollar costs quoted in Tables 4-1 to 4-4 with "typical" transport costs quoted about 10 years ago (actually 1976) of $25 \mathrm{~s} / \mathrm{kg}(13)$. If we take this $25 \mathrm{si} / \mathrm{kg}$ cost as an "average" number, then it is to be compared with the $17 \mathrm{\$} / \mathrm{kg}$ for the current "base case." The $25 \$ / \mathrm{kg}$ number needs to be adjusted for inflation. If we assume an inflation factor of 1.8, then cask shipping costs have dropped by about a factor of (1.8) (25)/17 $\sim 2.6$ over the past decade. The most likely cause for this reduction in costs is the fact that the fuel to be shipped now is cooler, the cask capacities much larger (cask capacities used to be about $1 / 2$ for truck casks and $7 / 17$ to $10 / 24$ for rail casks), and hence the number of trips less than half of what was expected in the mid-1970s.

The next set of cases, V, VI, and Cases VIIa, VIIb, and VIIC, all represent versions of intermodal transport (IMI). These cases were constructed to remain within the limits of existing site constraints. As one might expect, the cost trends here all tend to be up as more and more operations get to be involved. One reason why one might want to proceed with the IMT, nevertheless, is to reduce population dose, particularly the collective public radiation dose. This can be done by shifting progressively more to rail transport (see Chapter 5).

There are several nuances in the cost tabulations for IMT (Tab?es 4-4 to 4-9) that need to be pointed out. The general assumption has been made that transport campaigns cover a single large reactor discharge cycle of $\sim 35$ MTU. In IMT, where truck and rail transport are involved, an important variable is 
the number of casks loaded onto a single train. A sensitivity stuay was done to examine this situation for Case $V$, OWT[R] transport. If casks are kept idle at the railhead as more and more casks are loaded from truck to rail, the average cask utilization goes down and the average round-trip time will increase, hence a larger cask fleet will be needed. Thus, fleet capital costs wi il go up as will cask maintenance costs. However, more casks per train also imply fewer train trips. Fewer train trips mean lower hauling and security charges. The other costs, cask handling and development and certification costs, are essentially independent of the cask per train variable. In the case of IMT using OWT casks, the maximum number of casks shipped per train would be $\sim$ 15. Figure 4-1 shows the variation of the OWT $(R)$ cost component as a function of the number of casks/train. For this case, the net cost trend is down over the range of casks/train shown. (Similar trends could be expected for the LWT (R) IMT operation.)

For Cases $V$ and $V I$, we have assumed for purposes of our cost estimates that the casks loaded on a single train would be the maximum number of casks available per campaign. (This is about 25 LWT casks, 15 OWT casks, or 3 RR casks.) This results in a minimum cost case. In actuality, utility crews may on ly be available for about 10 days of single-shift operation (8). Such a crew could handle about 5 OWT casks, and thus the net costs for IMT transport would rise, as the number of casks per train are reduced (see Figure 4-1).

Another problem concerns the return of empty casks from the repository. In our calculations (and estimated costs) we have assumed that casks are returned via train, passing through the railhead as in forward transport. Another schei.e would be to return all LWT truck casks directly by truck. bypassing the transfer operation at the railhead. For this case, the costs would be somewhat lower than the quoted costs. For case VI, this cost differential is about $\$ 33$ million.

For IMT Cases VIIa to VIIC, there are fewer variations to be explored. In each case only three rail casks are transloaded, and the expectation is that three casks would indeed be handled in each operation. Also, these rail casks would all be returned by rail and thus regular truck transport is not involved. There is, however, still the option open to return the empty LWT casks considered in case VIIc by truck.

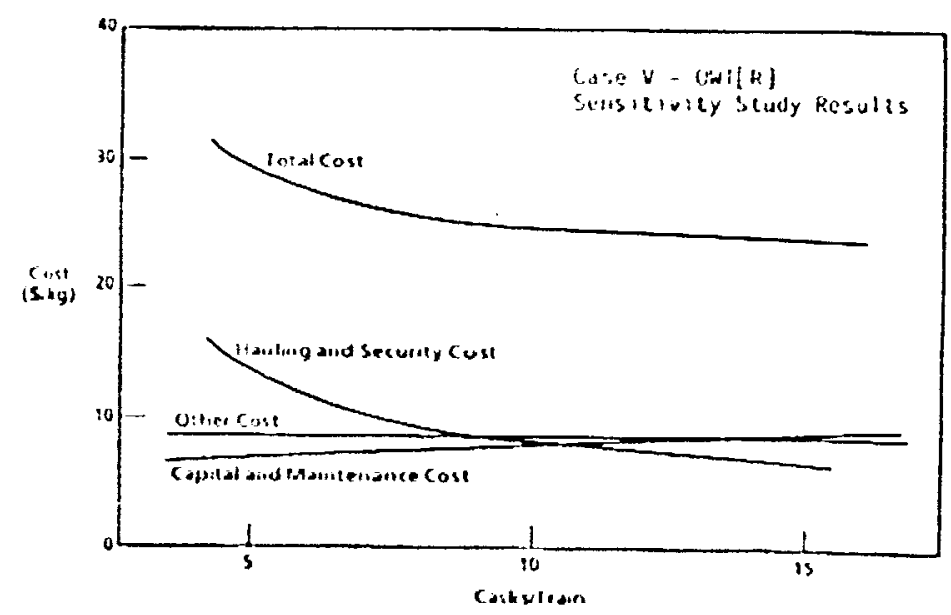

Figure 4-1. Cost Dependence on Casks/Train 
Table 4-1. Case I. All Casks Shipped by LWT

\begin{tabular}{lc}
\hline & LWT Cask $3 / 7$ \\
& Ship by Truck \\
\hline PWR/BWR MTU & $56503 / 30275$ \\
Total MTU & 86,779 \\
One-Way Distance (mi) & 2,210 \\
Average Speed Front Haul (mph) & 37.5 \\
Average Speed Back Haul (mph) & 37.5 \\
Development/Certification Cost & 6 \\
Cask Capital Cost & 98 \\
Hauling Cost & 722 \\
Maintenance Cost & 133 \\
Security Cost & 519 \\
Handling Cost & 575 \\
At Reactor & 325 \\
At Repository & 250 \\
Total LCC (mill \$) & $\$ 2,052$ \\
LCC per kgl & $\$ 24$ \\
PWR/BWR Cask Fleet & $39 / 22$ \\
Turnaround Time (days) & 8.4 \\
At Reactor & 2.0 \\
In-Transit & 4.9 \\
At Repository & 1.5 \\
Cask Availability (days) & 310 \\
Cask Round-Trips per Year & 36.9 \\
\hline \hline
\end{tabular}


Table 4-2. Case II. Reference Case: LWT/RR

\begin{tabular}{|c|c|c|c|}
\hline & $\begin{array}{l}\text { LWT Cask } 3 / 7 \\
\text { Ship by Truck }\end{array}$ & $\begin{array}{c}\text { Regular Rail } \\
\text { Cask } 21 / 48\end{array}$ & Total \\
\hline $\begin{array}{l}\text { PWR/BWR MTU } \\
\text { Total MTU } \\
\text { One-Way Distance (mi) } \\
\text { Average Speed Front Hau? (mph) } \\
\text { Average Speed Back Haul (mph) }\end{array}$ & $\begin{array}{l}24255 / 136 ! 7 \\
37,872 \\
2,333 \\
37.5 \\
37.5\end{array}$ & $\begin{array}{c}32248 / 16658 \\
48,906 \\
2,339 \\
11.85 \\
7.9\end{array}$ & 86,778 \\
\hline $\begin{array}{l}\text { Development/Certification Cost } \\
\text { Cask Capitai Cost } \\
\text { Hauling Cost } \\
\text { Maintenance Cost } \\
\text { Security Cost } \\
\text { Handling Cost } \\
\text { At Reactor } \\
\text { At Repository } \\
\text { Total (mill \$) }\end{array}$ & $\begin{array}{r}6 \\
43 \\
331 \\
59 \\
239 \\
251 \\
142 \\
109 \\
930\end{array}$ & $\begin{array}{r}8 \\
76 \\
258 \\
69 \\
23 \\
79 \\
44 \\
35 \\
514\end{array}$ & $\begin{array}{r}14 \\
119 \\
590 \\
128 \\
263 \\
331 \\
187 \\
144 \\
1,444\end{array}$ \\
\hline LCC per $\mathrm{KgU}$ & 25 & 11 & 17 \\
\hline PWR/BWR Cask Fleet & $17 / 10$ & $12 / 7$ & -- \\
\hline $\begin{array}{l}\text { Turnaround Time (days) } \\
\text { At Reactor } \\
\text { In-Transit } \\
\text { At Repository }\end{array}$ & $\begin{array}{l}8.7 \\
2.0 \\
5.2 \\
1.5\end{array}$ & $\begin{array}{r}28.7 \\
5.9 \\
20.6 \\
2.2\end{array}$ & $\begin{array}{l}-- \\
-- \\
--\end{array}$ \\
\hline Cask Availability (days) & 310 & 280 & -- \\
\hline Cask Round-Trips per Year & 35.7 & 9.8 & -- \\
\hline
\end{tabular}


Table 4-3. Case III. LWT, OWT, RR

\begin{tabular}{|c|c|c|c|c|}
\hline & $\begin{array}{l}\text { LWT Cask } \\
3 / 7 \\
\text { Ship by } \\
\text { Truck }\end{array}$ & $\begin{array}{l}\text { OWT Cask } \\
5 / 12 \\
\text { Ship by } \\
\text { Truck }\end{array}$ & $\begin{array}{l}\text { Regular Rail } \\
\text { Cask } 21 / 48\end{array}$ & Total \\
\hline $\begin{array}{l}\text { PWR/BWR MTU } \\
\text { Total MTU } \\
\text { One-Way Distance (mi) } \\
\text { Average Speed Front Haul (mph) } \\
\text { Average Speed Back Haul (mph) }\end{array}$ & $\begin{array}{l}5841 / 719 \\
6,560 \\
2,449 \\
37.5 \\
37.5\end{array}$ & $\begin{array}{c}18414 / 12899 \\
31,313 \\
2,309 \\
22.7 \\
22.7\end{array}$ & $\begin{array}{l}32248 / 16658 \\
48,906 \\
2,339 \\
11.85 \\
7.9\end{array}$ & $\begin{array}{c}86,779 \\
-- \\
-- \\
--\end{array}$ \\
\hline $\begin{array}{l}\text { Development/Certification Cost } \\
\text { Cask Capital Cost } \\
\text { Hauling Cost } \\
\text { Maintenance Cost } \\
\text { Security Cost } \\
\text { Handling Cost } \\
\text { At Reactor } \\
\text { At Repository } \\
\text { Total (mill \$) }\end{array}$ & $\begin{array}{r}6 \\
10 \\
59 \\
13 \\
43 \\
42 \\
24 \\
18 \\
173\end{array}$ & $\begin{array}{r}7 \\
38 \\
229 \\
47 \\
116 \\
130 \\
74 \\
\frac{57}{567}\end{array}$ & $\begin{array}{r}8 \\
76 \\
258 \\
59 \\
23 \\
79 \\
44 \\
\frac{35}{514}\end{array}$ & $\begin{array}{r}21 \\
124 \\
547 \\
129 \\
182 \\
252 \\
142 \\
110 \\
1,254\end{array}$ \\
\hline LCC per KgU. & 26 & 18 & 11 & 14 \\
\hline PWR/BWR Cask Fleet & $5 / 1$ & $11 / 8$ & $12 / 7$ & \\
\hline $\begin{array}{l}\text { Turnaround Time (days) } \\
\text { At Reactor } \\
\text { In-Transit } \\
\text { At Repository }\end{array}$ & $\begin{array}{l}8.9 \\
2.0 \\
5.4 \\
1.5\end{array}$ & $\begin{array}{r}12.0 \\
2.0 \\
8.5 \\
1.5\end{array}$ & $\begin{array}{r}28.7 \\
5.9 \\
20.6 \\
2.2\end{array}$ & \\
\hline Cask Availability (days) & 310 & 310 & 280 & \\
\hline Cask Round-Trips per Year & 34.7 & 25.9 & 9.8 & \\
\hline
\end{tabular}


Table 4-4. Case IV. LWT/OWT/RR/HRC

\begin{tabular}{|c|c|c|c|c|c|}
\hline . & $\begin{array}{l}\text { LWT Cask } 3 / 7 \\
\text { Ship by Truck }\end{array}$ & $\begin{array}{l}\text { OWT Cask } 5 / 12 \\
\text { Ship by Truck }\end{array}$ & $\begin{array}{l}\text { Regular Rail } \\
\text { Cask } 21 / 48\end{array}$ & $\begin{array}{l}\text { Hefty Rall } \\
24 / 60 \text { Cask }\end{array}$ & Total \\
\hline $\begin{array}{l}\text { PWR/BWR MTU } \\
\text { Total MTU } \\
\text { One-Way Distance (mi) } \\
\text { Average Speed Front Haul (mph) } \\
\text { Average Speed Back Haul (mph) }\end{array}$ & $\begin{array}{l}5841 / 719 \\
6,560 \\
2,449 \\
37.5 \\
37.5\end{array}$ & $\begin{array}{c}18414 / 12899 \\
31,313 \\
2,309 \\
22.7 \\
22.7\end{array}$ & $\begin{array}{c}5077 / 7989 \\
13,066 \\
2,360 \\
11.93 \\
7.96\end{array}$ & $\begin{array}{l}27171 / 8669 \\
35,840 \\
2,331 \\
11.86 \\
11.86\end{array}$ & $\begin{array}{c}56503 / 30275 \\
86,779 \\
-- \\
-- \\
--\end{array}$ \\
\hline $\begin{array}{l}\text { Development/Certification Cost: } \\
\text { Cask Capital Cost } \\
\text { Hauling Cost } \\
\text { Maintenance Cost } \\
\text { Security Cost } \\
\text { Hand ing Cost } \\
\text { At Reactor } \\
\text { At Repository } \\
\text { Total (mill \$) }\end{array}$ & $\begin{array}{r}6 \\
10 \\
59 \\
13 \\
43 \\
42 \\
24 \\
18 \\
\$ 173\end{array}$ & $\begin{array}{r}7 \\
38 \\
229 \\
47 \\
116 \\
130 \\
74 \\
57 \\
\$ 567\end{array}$ & $\begin{array}{r}8 \\
24 \\
71 \\
22 \\
6 \\
23 \\
13 \\
10 \\
\$ 154\end{array}$ & $\begin{array}{r}9 \\
55 \\
291 \\
48 \\
12 \\
50 \\
28 \\
22 \\
\$ 466\end{array}$ & $\begin{array}{r}30 \\
127 \\
650 \\
129 \\
178 \\
246 \\
138 \\
107 \\
\$ 1,360\end{array}$ \\
\hline LCC per $\mathrm{kgU}$ & $\$ 26$ & $\$ 18$ & $\$ 12$ & $\$ 13$ & $\$ 16$ \\
\hline PWR/BWR Cask F leet & $5 / 1$ & $11 / 8$ & $2 / 4$ & $8 / 3$ & -- \\
\hline $\begin{array}{l}\text { Turnaround Time (days) } \\
\text { At Reactor } \\
\text { In-Transit } \\
\text { At Repository }\end{array}$ & $\begin{array}{l}8.9 \\
2.0 \\
5.4 \\
1.5\end{array}$ & $\begin{array}{r}12.0 \\
2.0 \\
8.5 \\
1.5\end{array}$ & $\begin{array}{r}28.7 \\
5.9 \\
20.6 \\
2.2\end{array}$ & $\begin{array}{r}25.5 \\
6.9 \\
16.4 \\
2.2\end{array}$ & $\begin{array}{l}-- \\
-- \\
--\end{array}$ \\
\hline Cask Avallability (days) & 310 & 310 & 280 & 280 & - \\
\hline Cask Round-Trips per Year & 34.7 & 25.9 & 9.7 & 11.0 & -- \\
\hline
\end{tabular}


Table 4-5. Case V. LWT/OWT[R]/RR/HRC - Intermodal Transport

\begin{tabular}{|c|c|c|c|c|c|}
\hline & $\begin{array}{l}\text { LWT Cask } 3 / 7 \\
\text { Ship by Truck }\end{array}$ & $\begin{array}{l}\text { OWT Cask } 5 / 12 \\
\text { Ship by Rail }\end{array}$ & $\begin{array}{c}\text { Regular Rafi } \\
\text { Cask } 21 / 48\end{array}$ & $\begin{array}{l}\text { Hefty Ra11 } \\
24 / 60 \text { Cask }\end{array}$ & Total \\
\hline $\begin{array}{l}\text { PWR/BWR MTU } \\
\text { Total MTU } \\
\text { one-Way Distance (m1) } \\
\text { Average Speed Front Haul (mph) } \\
\text { Average Speed Back Haul (mph) }\end{array}$ & $\begin{array}{l}5841 / 719 \\
6.560 \\
2,449 \\
37.5 \\
37.5\end{array}$ & $\begin{array}{c}18414 / 12899 \\
31.313 \\
2,475 \\
12.25 \\
8.17\end{array}$ & $\begin{array}{l}5077 / 7989 \\
13,066 \\
2,360 \\
11.93 \\
7.96\end{array}$ & $\begin{array}{l}27171 / 8669 \\
35,840 \\
2,331 \\
11.86 \\
11.86\end{array}$ & $\begin{array}{c}56503 / 30275 \\
86,779 \\
\cdots \\
\cdots \\
\cdots\end{array}$ \\
\hline $\begin{array}{l}\text { Development/Certification Cost } \\
\text { Cask Capital Cost } \\
\text { Hauling Cost } \\
\text { Maintenance Cost } \\
\text { Security Cost } \\
\text { Handling Cost } \\
\text { At Reactor } \\
\text { At Repository } \\
\text { Intermodal Transfer Cost } \\
\text { Total (mill \$) }\end{array}$ & $\begin{array}{r}6 \\
10 \\
59 \\
13 \\
43 \\
42 \\
24 \\
18 \\
0 \\
173\end{array}$ & $\begin{array}{r}7 \\
126 \\
182 \\
155 \\
13 \\
130 \\
74 \\
57 \\
138 \\
\$ 751\end{array}$ & $\begin{array}{r}8 \\
24 \\
71 \\
22 \\
6 \\
23 \\
13 \\
10 \\
0 \\
\$ 154\end{array}$ & $\begin{array}{r}9 \\
55 \\
291 \\
48 \\
12 \\
50 \\
28 \\
22 \\
0 \\
466\end{array}$ & $\begin{array}{r}30 \\
215 \\
604 \\
238 \\
75 \\
246 \\
138 \\
107 \\
138 \\
1,544\end{array}$ \\
\hline LCC per $\mathrm{KgU}$ & $\$ 26$ & $\$ 24$ & $\$ 12$ & $\$ 13$ & $\$ 18$ \\
\hline PWR/BWR Cask Fleet & $5 / 1$ & $37 / 26$ & $2 / 4$ & $8 / 3$ & -- \\
\hline $\begin{array}{l}\text { Turnaround Time (days) } \\
\text { At Reactor } \\
\text { In-Transit } \\
\text { Intermodal Transfer } \\
\text { At Repository }\end{array}$ & $\begin{array}{l}8.9 \\
2.0 \\
5.4 \\
-1.5 \\
1.5\end{array}$ & $\begin{array}{r}40.5 \\
16.0 \\
21.0 \\
2.0 \\
1.5\end{array}$ & $\begin{array}{r}28.7 \\
5.9 \\
20.6 \\
-- \\
2.2\end{array}$ & $\begin{array}{r}25.5 \\
6.9 \\
16.4 \\
--. \\
2.2\end{array}$ & $\begin{array}{l}-- \\
-- \\
-- \\
-\end{array}$ \\
\hline Cask Availability (days) & 310 & 310 & 280 & 280 & -- \\
\hline Cask Round-Trips per Year & 34.7 & 7.6 & 9.7 & 11.0 & -- \\
\hline
\end{tabular}


Table 1-6. Case VI. LWT/R]/OWT[R]/RR/HRC - Intermodal Transport

\begin{tabular}{|c|c|c|c|c|c|}
\hline & $\begin{array}{l}\text { LWT Cask } 3 / 7 \\
\text { Ship by Rail }\end{array}$ & $\begin{array}{l}\text { OWT Cast } 5 / 12 \\
\text { Ship by Rafl }\end{array}$ & $\begin{array}{l}\text { Reyular Rafl } \\
\text { Cesk 21/48 }\end{array}$ & $\begin{array}{l}\text { Hefty Ra11 } \\
24 / 60 \text { Cask }\end{array}$ & Total \\
\hline $\begin{array}{l}\text { PWR/BWR MTU } \\
\text { Total MTU } \\
\text { One-Way Distance (mi) } \\
\text { Average Speed Front Haul (mph) } \\
\text { Average Speed Back Haul (mph) }\end{array}$ & $\begin{array}{l}5841 / 719 \\
6,560 \\
2,592 \\
12.56 \\
8.37\end{array}$ & $\begin{array}{c}18414 / 12899 \\
31,313 \\
2,475 \\
12.25 \\
8.17\end{array}$ & $\begin{array}{l}5077 / 7989 \\
13,066 \\
2,360 \\
11.93 \\
7.96\end{array}$ & $\begin{array}{l}27171 / 8669 \\
35,840 \\
2,331 \\
11.86 \\
11.86\end{array}$ & $\begin{array}{c}56503 / 30275 \\
86,779 \\
\cdots \\
-- \\
--\end{array}$ \\
\hline $\begin{array}{l}\text { Deve lopment/Certification Cost } \\
\text { Cask Capital Cost } \\
\text { Hauling Cost } \\
\text { Maintenance Cost } \\
\text { Security Cost } \\
\text { Handling Cost } \\
\text { At Reactor } \\
\text { At Repository } \\
\text { Intermodal Transfer Cost } \\
\text { Total (mill } \$ \text { ) }\end{array}$ & $\begin{array}{r}6 \\
46 \\
42 \\
63 \\
3 \\
42 \\
24 \\
18 \\
46 \\
\$ 248\end{array}$ & $\begin{array}{r}7 \\
126 \\
182 \\
155 \\
13 \\
130 \\
74 \\
57 \\
138 \\
\$ 751\end{array}$ & $\begin{array}{r}8 \\
24 \\
71 \\
22 \\
6 \\
23 \\
13 \\
10 \\
0 \\
\$ 154\end{array}$ & $\begin{array}{r}9 \\
55 \\
291 \\
48 \\
12 \\
50 \\
28 \\
22 \\
0 \\
\$ 466\end{array}$ & $\begin{array}{r}30 \\
251 \\
587 \\
288 \\
34 \\
246 \\
138 \\
107 \\
184 \\
\$ 1,619\end{array}$ \\
\hline LCC per KgU & $\$ 38$ & $\$ 24$ & $\$ 12$ & $\$ 13$ & $\$ 19$ \\
\hline PWR/BWR Cask Fleet & $25 / 4$ & $37 / 26$ & $2 / 4$ & $8 / 3$ & -- \\
\hline $\begin{array}{l}\text { Turnaround Time (days) } \\
\text { At Reactor } \\
\text { In-Transit } \\
\text { Intermodal Transfer } \\
\text { At Repository }\end{array}$ & $\begin{array}{r}51.0 \\
26.0 \\
21.5 \\
2.0 \\
1.5\end{array}$ & $\begin{array}{r}40.5 \\
16.0 \\
21.0 \\
2.0 \\
1.5\end{array}$ & $\begin{array}{r}28.7 \\
5.9 \\
20.6 \\
-- \\
2.2\end{array}$ & $\begin{array}{r}25.5 \\
6.9 \\
16.4 \\
-.- \\
2.2\end{array}$ & $\begin{array}{l}-- \\
-- \\
-- \\
--\end{array}$ \\
\hline Cask Availability (days) & 310 & 310 & 280 & 280 & -- \\
\hline Cask Round-Trips per Year & 6.1 & 7.6 & 9.7 & 11.0 & -- \\
\hline
\end{tabular}


Table 4-7. Case VIIa. LWT/RES. OWT/HHTIR]/RR/HRC - Intermodal Transport

\begin{tabular}{|c|c|c|c|c|c|c|}
\hline & $\begin{array}{l}\text { LWT Cask } \\
3 / 7 \\
\text { Ship by } \\
\text { Truck }\end{array}$ & $\begin{array}{c}\text { HHT by } \\
\text { Rafl } 21 / 48\end{array}$ & $\begin{array}{l}\text { Residual OWT } \\
\text { by } 5 / 12 \text { Truck }\end{array}$ & $\begin{array}{l}\text { Hefty Rail } \\
24 / 60 \text { Cask }\end{array}$ & $\begin{array}{c}\text { Regular Rafl } \\
\text { Cask } 21 / 48\end{array}$ & Total \\
\hline $\begin{array}{l}\text { PWR/BWR MTU } \\
\text { Tota) MTU } \\
\text { One-Way Distance (mi) } \\
\text { Average Speed Front Haul (mph) } \\
\text { Average Speed Back Haul (mph) }\end{array}$ & $\begin{array}{l}5841 / 719 \\
6,560 \\
2,449 \\
37.5 \\
37.5\end{array}$ & $\begin{array}{l}11892 / 7722 \\
19,614 \\
2,473 \\
12.24 \\
8.16\end{array}$ & $\begin{array}{c}6522 / 5176 \\
11,698 \\
2,286 \\
22.7 \\
22.7\end{array}$ & $\begin{array}{l}27171 / 8669 \\
35,840 \\
2,331 \\
11.86 \\
11.86\end{array}$ & $\begin{array}{l}5077 / 7989 \\
13,066 \\
2,360 \\
11.93 \\
7.96\end{array}$ & $\begin{array}{c}56503 / 30275 \\
86,778 \\
-- \\
-- \\
--\end{array}$ \\
\hline $\begin{array}{l}\text { Developmerit/Certiflcat lon Cost } \\
\text { Cask Capital Cost } \\
\text { Hauling Cost } \\
\text { Maintenance Cost } \\
\text { Security Cost } \\
\text { Handling Cost } \\
\text { At Reactor } \\
\text { At Repository } \\
\text { Intermodal Transfer Cost } \\
\text { Total LCC (mill \$) }\end{array}$ & $\begin{array}{r}6 \\
10 \\
59 \\
13 \\
43 \\
42 \\
24 \\
18 \\
0 \\
\$ 173\end{array}$ & $\begin{array}{r}8 \\
36 \\
109 \\
33 \\
10 \\
32 \\
18 \\
14 \\
94 \\
\$ 323\end{array}$ & $\begin{array}{r}7 \\
16 \\
85 \\
20 \\
43 \\
49 \\
28 \\
21 \\
0 \\
\$ 219\end{array}$ & $\begin{array}{r}9 \\
55 \\
291 \\
48 \\
12 \\
50 \\
28 \\
22 \\
0 \\
\$ 466\end{array}$ & $\begin{array}{r}8 \\
24 \\
71 \\
22 \\
6 \\
23 \\
13 \\
10 \\
0 \\
\$ 154\end{array}$ & $\begin{array}{r}38 \\
141 \\
615 \\
135 \\
137 \\
197 \\
111 \\
86 \\
94 \\
\$ 1,356\end{array}$ \\
\hline LCC per KgU & $\$ 2.6$ & $\$ 16$ & $\$ 19$ & $\$ 13$ & $\$ 12$ & $\$ 16$ \\
\hline PWR/BWR Cask Fleet & $5 / 1$ & $5 / 4$ & $4 / 4$ & $8 / 3$ & $2 / 4$ & -- \\
\hline $\begin{array}{l}\text { Turnaround Time (daye) } \\
\text { At Reactor } \\
\text { Intermodal Transfer } \\
\text { In-Transit } \\
\text { At Repository }\end{array}$ & $\begin{array}{l}8.9 \\
2.0 \\
0.0 \\
5.4 \\
1.5\end{array}$ & $\begin{array}{r}33.2 \\
5.9 \\
4.0 \\
21.0 \\
2.2\end{array}$ & $\begin{array}{l}11.9 \\
2 \\
0.0 \\
8 \\
2\end{array}$ & $\begin{array}{r}25.5 \\
6.9 \\
0.0 \\
16.4 \\
2.2\end{array}$ & $\begin{array}{r}28.7 \\
5.9 \\
0.0 \\
20.6 \\
2.2\end{array}$ & $\begin{array}{l}-- \\
-- \\
-- \\
--\end{array}$ \\
\hline Cask Availability (days) & 310 & 280 & 310 & 280 & 280 & - \\
\hline Cask Round-Trips per Year & 34.7 & 8.4 & 26.1 & 11.0 & 9.7 & -- \\
\hline
\end{tabular}


lable 4-8. Case VIIb. LWT/RES. OWTIR|/HHT|R|/RR/HRC - Intermodal Transport

\begin{tabular}{|c|c|c|c|c|c|c|}
\hline & $\begin{array}{l}\text { I.WT Cask } \\
3 / 7 \\
\text { Ship by } \\
\text { Truck }\end{array}$ & $\begin{array}{c}\text { HHT by } \\
\text { Rafl } 21 / 48\end{array}$ & $\begin{array}{l}\text { Residual OWT } \\
\text { by } 5 / 12 \text { Ra } 11\end{array}$ & $\begin{array}{l}\text { Hefty Rail } \\
24 / 60 \text { Cask }\end{array}$ & $\begin{array}{l}\text { Regular Rail } \\
\text { Cask } 21 / 48\end{array}$ & Total \\
\hline $\begin{array}{l}\text { PWR/BWR MTU } \\
\text { Total MTU } \\
\text { One-Way Distance (mi) } \\
\text { Average Speed Front Haul (mph) } \\
\text { Average Speed Back Haul (mph) }\end{array}$ & $\begin{array}{l}5841 / 719 \\
6,560 \\
2,449 \\
37.5 \\
37.5\end{array}$ & $\begin{array}{r}11892 / 7721 \\
19,613 \\
2,473 \\
12.24 \\
8.16\end{array}$ & $\begin{array}{l}6522 / 5176 \\
11,698 \\
2,478 \\
12.26 \\
8.17\end{array}$ & $\begin{array}{l}27171 / 8669 \\
35,840 \\
2,331 \\
11.86 \\
11.86\end{array}$ & $\begin{array}{l}5077 / 7989 \\
13,066 \\
2,360 \\
11.93 \\
7.96\end{array}$ & $\begin{array}{c}56503 / 30275 \\
86,777 \\
-- \\
-- \\
-\end{array}$ \\
\hline $\begin{array}{l}\text { Deve lopment/Certification Cost } \\
\text { Cask Capital Cost } \\
\text { Hauling Cost } \\
\text { Maintenance Cost } \\
\text { Security Cost } \\
\text { Handling Cost } \\
\text { At Reactor } \\
\text { At Repository } \\
\text { Intermodal Transfer Cost } \\
\text { Total LCC (mill \$) }\end{array}$ & $\begin{array}{r}6 \\
10 \\
59 \\
13 \\
43 \\
42 \\
24 \\
18 \\
0 \\
\$ 173\end{array}$ & $\begin{array}{r}8 \\
36 \\
109 \\
33 \\
10 \\
32 \\
18 \\
14 \\
94 \\
\$ 323\end{array}$ & $\begin{array}{r}7 \\
48 \\
68 \\
59 \\
5 \\
49 \\
28 \\
21 \\
51 \\
\$ 287\end{array}$ & $\begin{array}{r}9 \\
55 \\
291 \\
48 \\
12 \\
50 \\
28 \\
22 \\
0 \\
\$ 466\end{array}$ & $\begin{array}{r}8 \\
24 \\
71 \\
22 \\
6 \\
23 \\
13 \\
10 \\
0 \\
\$ 154\end{array}$ & $\begin{array}{r}38 \\
173 \\
599 \\
174 \\
77 \\
197 \\
111 \\
86 \\
146 \\
\$ 1,403\end{array}$ \\
\hline LCC per KgJ & $\$ 26$ & $\$ 16$ & $\$ 25$ & $\$ 13$ & $\$ 12$ & $\$ 16$ \\
\hline PWR/BWR Cask Fleet & $5 / 1$ & $5 / 4$ & $13 / 11$ & $8 / 3$ & $2 / 4$ & - \\
\hline $\begin{array}{l}\text { Turnaround Time (days) } \\
\text { At Reactor } \\
\text { Intermodal Transfer } \\
\text { In-Transit } \\
\text { At Repository }\end{array}$ & $\begin{array}{l}8.9 \\
2.0 \\
-- \\
5.4 \\
1.5\end{array}$ & $\begin{array}{r}33.2 \\
5.9 \\
4.0 \\
21.0 \\
2.2\end{array}$ & $\begin{array}{r}40.6 \\
16.0 \\
2.0 \\
21.1 \\
1.5\end{array}$ & $\begin{array}{r}25.5 \\
6.9 \\
-- \\
16.4 \\
2.2\end{array}$ & $\begin{array}{r}28.7 \\
5.9 \\
-- \\
20.6 \\
2.2\end{array}$ & $\begin{array}{l}m \\
-- \\
m \\
--\end{array}$ \\
\hline Cask Availability (days) & 310 & 280 & 310 & 280 & 280 & -- \\
\hline Cask Round-Trips per Year & 34.7 & 8.4 & 7.6 & 11.0 & 9.7 & -- \\
\hline
\end{tabular}


Table 4-9. Case VIIC. LWT[R]/RES. OWT[R]/HHT/R]/RR/HRC - Intermodal Transport

\begin{tabular}{|c|c|c|c|c|c|c|}
\hline & $\begin{array}{l}\text { L.WT Cask } \\
3 / 7 \\
\text { Ship by } \\
\text { Rail }\end{array}$ & $\begin{array}{c}\text { HHT by } \\
\text { Rail } 21 / 48\end{array}$ & $\begin{array}{l}\text { Residual OWT } \\
\text { by } 5 / 12 \text { Rail }\end{array}$ & $\begin{array}{l}\text { Hefty Rail } \\
24 / 60 \text { Cask }\end{array}$ & $\begin{array}{c}\text { Regular Raill } \\
\text { Cask } 21 / 48\end{array}$ & Total \\
\hline $\begin{array}{l}\text { PWR/BWR MTU } \\
\text { Total MTU } \\
\text { One-Way Distance (mi) } \\
\text { Average Speed Front Haul (mph) } \\
\text { Average Speed Back Haul (mph) }\end{array}$ & $\begin{array}{c}5841 / 719 \\
6,560 \\
2,449 \\
37.5 \\
37.5\end{array}$ & $\begin{array}{c}11892 / 7721 \\
19.613 \\
2,473 \\
12.24 \\
8.16\end{array}$ & $\begin{array}{l}6522 / 5176 \\
11,698 \\
2,478 \\
12.26 \\
8.17\end{array}$ & $\begin{array}{c}27171 / 8669 \\
35,840 \\
2,331 \\
11.86 \\
11.86\end{array}$ & $\begin{array}{c}5077 / 7989 \\
13,066 \\
2,360 \\
11.93 \\
7.96\end{array}$ & $\begin{array}{c}56503 / 30275 \\
86.777 \\
-- \\
-- \\
--\end{array}$ \\
\hline $\begin{array}{l}\text { Deve lopment/Certification Cost } \\
\text { Cask Capital Cost } \\
\text { Hauling Cost } \\
\text { Maintenance Cost } \\
\text { Security Cost } \\
\text { Handling Cost } \\
\text { At Reactor } \\
\text { At Repository } \\
\text { Intermoda) Transfer Cost } \\
\text { Tota) LCC (mill \$) }\end{array}$ & $\begin{array}{r}6 \\
46 \\
42 \\
63 \\
3 \\
42 \\
24 \\
18 \\
46 \\
\$ 248\end{array}$ & $\begin{array}{r}8 \\
36 \\
109 \\
33 \\
10 \\
32 \\
18 \\
14 \\
94 \\
\$ 323\end{array}$ & $\begin{array}{r}7 \\
48 \\
68 \\
59 \\
5 \\
49 \\
28 \\
21 \\
51 \\
\$ 287\end{array}$ & $\begin{array}{r}9 \\
55 \\
291 \\
48 \\
12 \\
50 \\
28 \\
22 \\
0 \\
\$ 466\end{array}$ & $\begin{array}{r}8 \\
24 \\
71 \\
22 \\
6 \\
23 \\
13 \\
10 \\
0 \\
\$ 154\end{array}$ & $\begin{array}{r}38 \\
209 \\
582 \\
224 \\
37 \\
197 \\
111 \\
86 \\
192 \\
\$ 1,478\end{array}$ \\
\hline LCC per $\mathrm{KgU}$ & $\$ 38$ & $\$ 16$ & $\$ 25$ & $\$ 13$ & $\$ 12$ & $\$ 17$ \\
\hline PWR/BWR Cask Fleet & $25 / 4$ & $5 / 4$ & $13 / 11$ & $8 / 3$ & $2 / 4$ & -- \\
\hline $\begin{array}{l}\text { Turnaround Time (days) } \\
\text { At Reactor } \\
\text { Intermodal Transfer } \\
\text { In-Transit } \\
\text { At Repository }\end{array}$ & $\begin{array}{r}51.0 \\
26.0 \\
2.0 \\
21.5 \\
1.5\end{array}$ & $\begin{array}{r}33.2 \\
5.9 \\
4.0 \\
21.0 \\
2.2\end{array}$ & $\begin{array}{r}40.6 \\
16.0 \\
2.0 \\
21.1 \\
1.5\end{array}$ & $\begin{array}{r}25.5 \\
6.9 \\
-- \\
16.4 \\
2.2\end{array}$ & $\begin{array}{r}28.7 \\
5.9 \\
-- \\
20.6 \\
2.2\end{array}$ & $\begin{array}{l}-- \\
-- \\
-- \\
-- \\
--\end{array}$ \\
\hline Cask Availability (days) & 310 & 280 & 310 & 280 & 280 & -- \\
\hline Cask Round-Trips per Year & 6.1 & 8.4 & 7.6 & 11.0 & 9.7 & -- \\
\hline
\end{tabular}




\subsection{LIFE-CYCLE DOSE (LCD) ANALYSES}

The life-cycle dose (LCD) analyses were carried out as described in Section 3.3 of this report. The results for the nine cases and subcases are presented in Tables 5-1 to 5-9.

The information presented in the tables does not only include the LCD results, but also some basic input data for each case, i.e., life-time metric tons of uranium (MTU) discharged, number of reactor plants involved, and weighted-average distances from the plants to the repository.

The results are given in terms of total and public person-rem for each case, as well as in person-rem/MTU. Several points are worth noting about these results. As to be expected, the truck doses are considerably higher than the rail doses, by about a factor of 10(!). For instance, in case II we find the truck dose to be 1.1 person-rem/MTU, compared to a rail dose of about 0.12 person-rem/MTU. When both truck and rail shipments are used together, the combined dose for Case II is about 0.53 person-rem/MTU. (A similar calculation made in the PNL ALARA report for 30/70 truck/rail and 60/40 PWR/BWR split assumptions gives a combined dose of 0.50 person-rem/MTU.)

If all spent nuclear fuel (SNF) casks were shipped by legal-weight truck (LWT) (Case I)--a rather contrived bounding case--the total dose would be about 1.0 person-rem/MTU* and the public dose would be 0.42 person-rem/MTU. For this extreme case, the total population dose is close to 100,000 person-rem. Assuming 200 latent cancer fatalities (LCFs) per $10^{6}$ person-rem (14), this 100,000 person-rem dose would result in about 20 LCFs related to radioactive exposure from normal transport operations. For the base case, which involves a substantial rail shipment fraction, the fatality rate would be about half of that figure.

In Cases III and IV, the effect of using large-capacity casks, i.e., overweight truck casks (OWTCS) and hefty rail casks (HRCS), wherever possible, is examined. For example, using OWTCs (Case III) in truck transport whenever feasible within reactor crane limitations, reduces the total population dose from 0.53 (Case II) to 0.39 person-rem/MTU (Case III). The public dose is reduced from 0.20 (Case II) to 0.15 person-rem/MTU.

The remaining cases $V$ through VII represent intermodal transport (IMT). Transshipping the OWTCs by rail results in a fairly large dose reduction percentage-wise, i.e., the population dose drops from 0.39 person-rem/MTU (for Case IV [OWTCs shipped by rail]), to 0.27 person-rem/MTU (for Case V. 30\% decrease). The public dose drops to 0.045 person-rem/MTU from 0.15 person-rem/MTU.

If all fuel is shipped by rail (Case VI), such that both OWTCs and LWTCS are transferred cnto railcars, this "all rail" dose is 0.23 person-rem/MTU with a corresponding public dose of 0.013 person-rem/MTU.

The reason that the truck dose is 1.0 for this case and 1.1 for case II is iraceable to the different ractors involved in Case I and Case II. 
Finally, Cases VIIa, $b$, and $c$ represent another set of long-distance rail shipments. In Case VII, heavy-haul trucks are used to haul 100-ton casks to the nearest raithead from truck-designated reactors with crane capacities of 100 tons or more but no direct rail access. Case VIIC, which is the final "all rail " transport version, ends up with a total dose of 0.18 person-rem/MTU and a publ ic dose of 0.010 person-rem/MTU.

A public dose level of 0.010 person-rem/MTU is quite small. For a shipment of $\sim 100,000$ MTU, this amounts to 1,000 person-rem corresponding to a public LCF rate ascribable to nuclear waste transport of 0.2 . It is clear that from this public risk point of view, rail transport may be a preferred waste transport mode. 


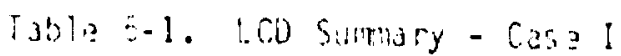

Cask Transport bia !WT

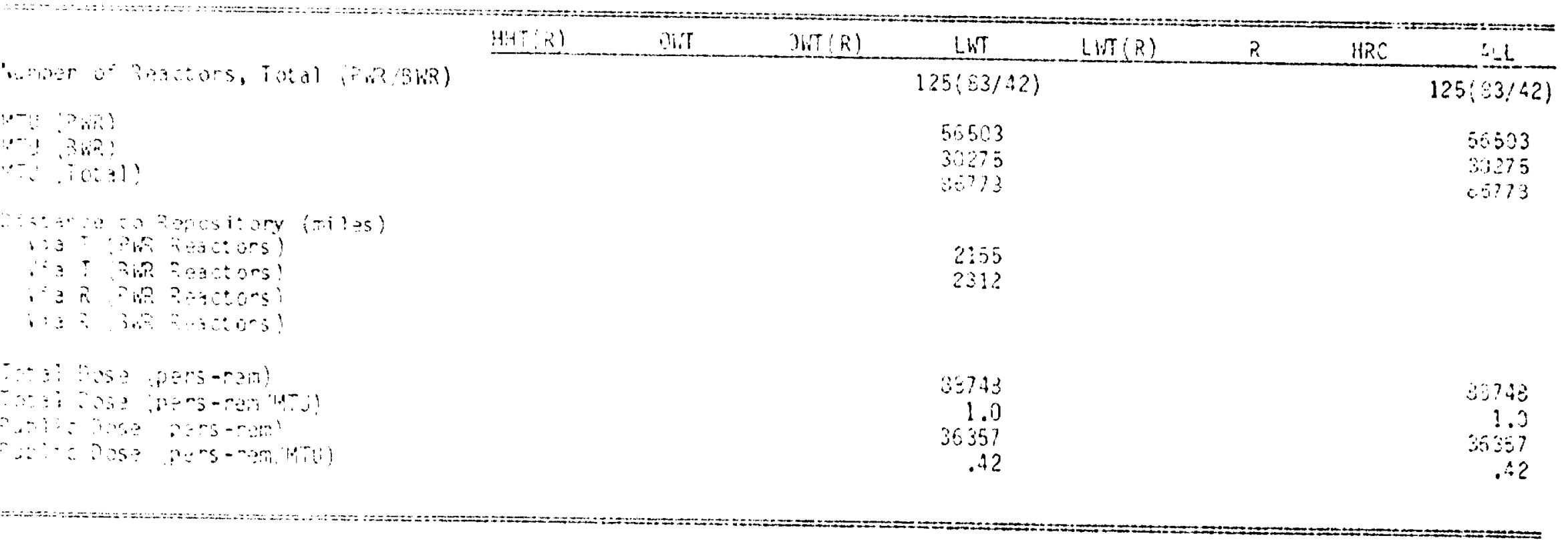


Table 5-2. (1.) Sumbery - Case II

Cask Transport Via l.wi/R

\begin{tabular}{|c|c|c|c|c|}
\hline & $2 \pi \quad 2 n T(R)$ & $L W T(R)$ & $R$ & HiR \\
\hline 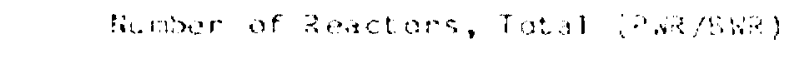 & & $51(10) 722)$ & $64(43: 21)$ & $125(\{33 / 42)$ \\
\hline 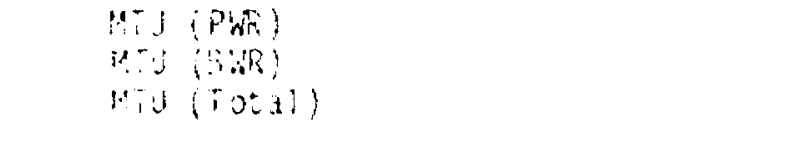 & & $\begin{array}{l}24255 \\
13615 \\
3 ? 371\end{array}$ & $\begin{array}{l}32248 \\
10658 \\
12300\end{array}$ & $\begin{array}{l}53503 \\
31274 \\
85777\end{array}$ \\
\hline 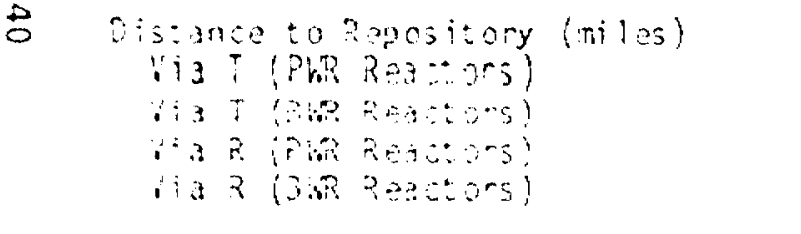 & & $\begin{array}{l}23.21 \\
2353\end{array}$ & $\begin{array}{l}2258 \\
2455\end{array}$ & \\
\hline 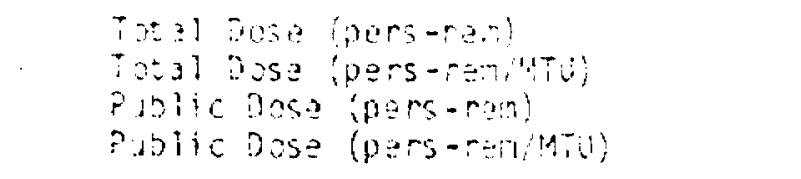 & & $\begin{array}{r}40.33 \\
137.17 \\
.44\end{array}$ & $\begin{array}{r}5514 \\
.12 \\
249 \\
.0051\end{array}$ & $\begin{array}{r}45547 \\
.53 \\
15035 \\
.20\end{array}$ \\
\hline
\end{tabular}


Table 5-3. LCD Surmary - Case ill

Cask Traisport Via 0 T/Lin/R

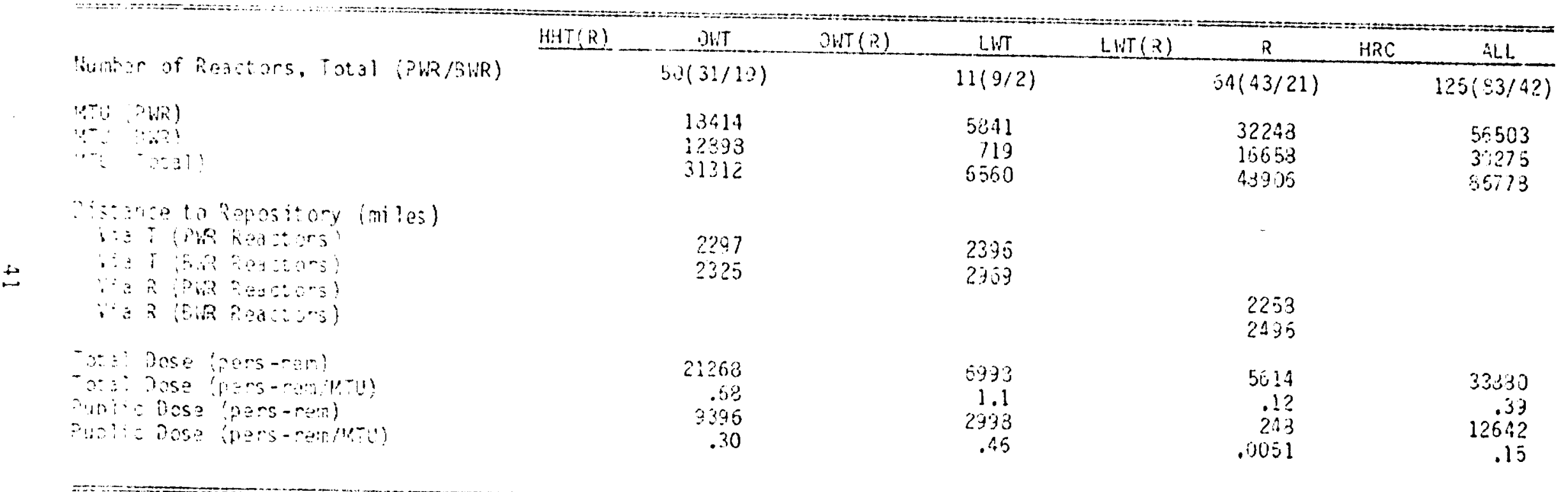


Table 5-1. LCD Summary - Case IV

Cask Transport Via OWT/LinT/R/HRC

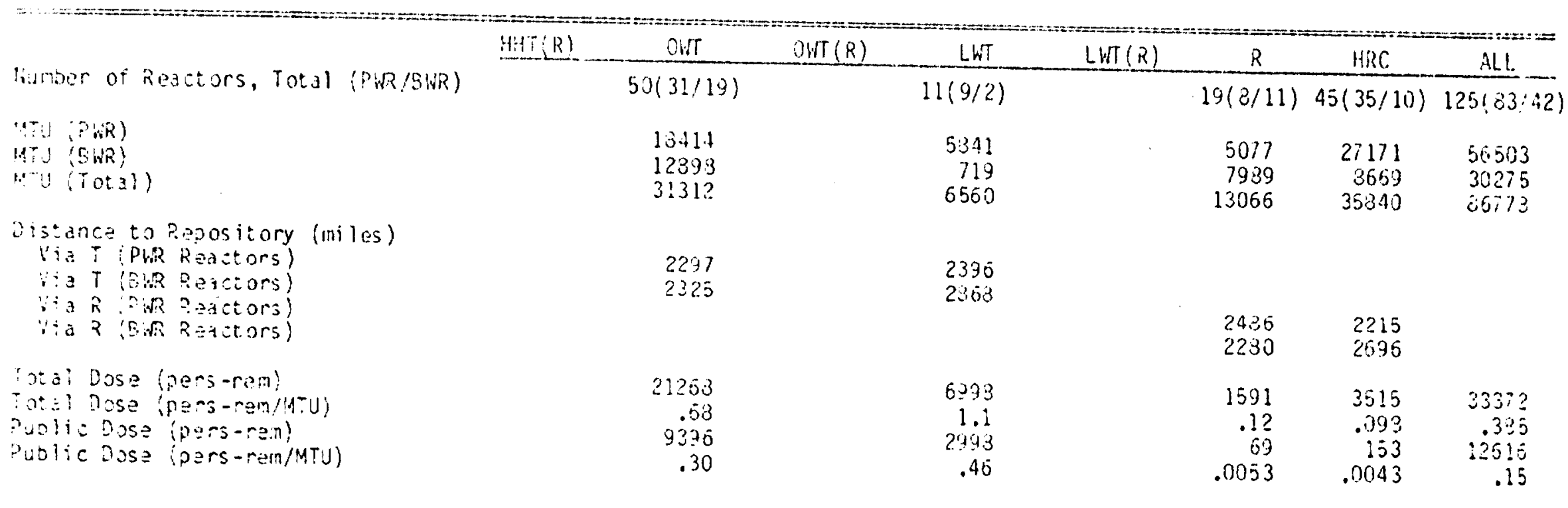

= 
Table 5-5. LCD Sumnary - Case V

Cask Transport Via OWT(R)/L.WT/R/HRC

\begin{tabular}{|c|c|c|c|c|c|c|c|}
\hline & $M H T(R)$ & DWT $(R)$ & LWT & $L W T(R)$ & R & HRC & $A L L$ \\
\hline :unber of reactors, Total (PWR/BWR) & & $50(31 / 19)$ & $11 / 9 / 2)$ & & $19(8 / 11)$ & $45(35 / 10)$ & $125(83 / 42)$ \\
\hline $\begin{array}{l}\text { MTU (PUR) } \\
\text { ATy (3in) } \\
\text { gut (Total) }\end{array}$ & & $\begin{array}{l}18414 \\
12898 \\
31312\end{array}$ & $\begin{array}{r}5841 \\
719 \\
6560\end{array}$ & & $\begin{array}{r}5077 \\
7989 \\
13066\end{array}$ & $\begin{array}{r}27171 \\
8669 \\
35340\end{array}$ & $\begin{array}{l}56503 \\
30275 \\
86778\end{array}$ \\
\hline 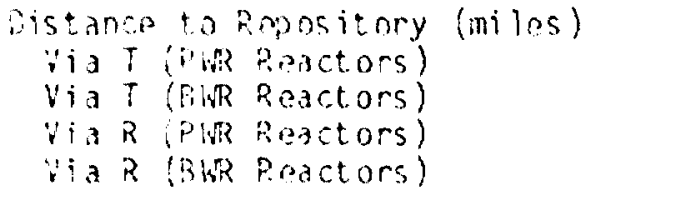 & & $\begin{array}{l}2404 \\
2490\end{array}$ & $\begin{array}{l}2396 \\
2868\end{array}$ & & $\begin{array}{l}2486 \\
2230\end{array}$ & $\begin{array}{l}2215 \\
2696\end{array}$ & \\
\hline 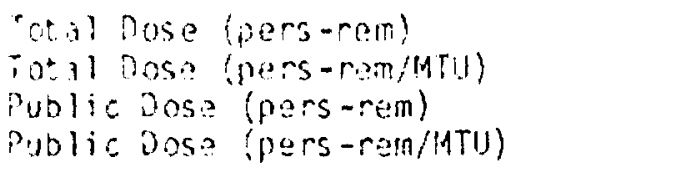 & & $\begin{array}{r}11141 \\
.36 \\
694 \\
.022\end{array}$ & $\begin{array}{r}6998 \\
1.1 \\
2998 \\
.46\end{array}$ & & $\begin{array}{r}1591 \\
.12 \\
69 \\
.0053\end{array}$ & $\begin{array}{r}3515 \\
.098 \\
153 \\
.0043\end{array}$ & $\begin{array}{r}23245 \\
.27 \\
3914 \\
.045\end{array}$ \\
\hline
\end{tabular}


Table 5-6. LCD Summary - Case VI

Cask Transport Via OWT(R)/LWT(R)/R/HRC

\begin{tabular}{|c|c|c|c|c|c|c|c|c|}
\hline & HHT $(R)$ & OWT & $O W T(R)$ & LWT & $L W T(R)$ & $\mathrm{R}$ & $\mathrm{HRC}$ & ALL \\
\hline Whather of Reactors, Total (PWR/BWR) & & & $50 / 31 / 29$ & & $11(9 / 2)$ & $19(8 / 11)$ & $45(35 / 10)$ & $125(83 / 42)$ \\
\hline $\begin{array}{l}\text { MUU (PWR) } \\
\text { WTU (BWR) } \\
\text { WTU (Total) }\end{array}$ & & & $\begin{array}{l}13414 \\
12898 \\
31312\end{array}$ & & $\begin{array}{r}5841 \\
719 \\
6560\end{array}$ & $\begin{array}{r}5077 \\
7989 \\
13066\end{array}$ & $\begin{array}{r}27171 \\
8669 \\
35840\end{array}$ & $\begin{array}{l}56503 \\
30275 \\
36778\end{array}$ \\
\hline 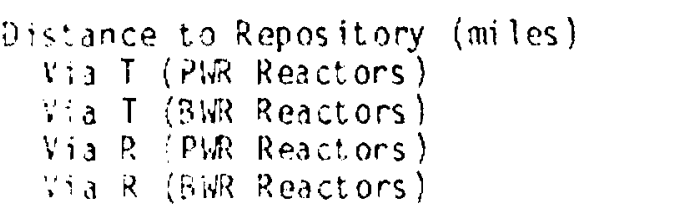 & & & $\begin{array}{l}2464 \\
2490\end{array}$ & & $\begin{array}{l}2525 \\
3129\end{array}$ & $\begin{array}{l}2436 \\
2280\end{array}$ & $\begin{array}{l}2215 \\
2696\end{array}$ & \\
\hline $\begin{array}{l}\text { ot a) Dose (pers-rem) } \\
\text { Total Dose (pers-rem/MTU) } \\
\text { Public Dose (pers-rem) } \\
\text { Pithlic Dose (pers-rem/HTU) }\end{array}$ & & & $\begin{array}{r}11141 \\
.36 \\
694 \\
.022\end{array}$ & & $\begin{array}{r}3778 \\
.58 \\
254 \\
.039\end{array}$ & $\begin{array}{r}1591 \\
.12 \\
69 \\
.0053\end{array}$ & $\begin{array}{r}3515 \\
.099 \\
153 \\
.0043\end{array}$ & $\begin{array}{r}20025 \\
.23 \\
1170 \\
.013\end{array}$ \\
\hline
\end{tabular}


Table 5-7. LCD Summary - Case VIla

Cask Transport Via HHT(R),ONT,LWT/R, /HRC

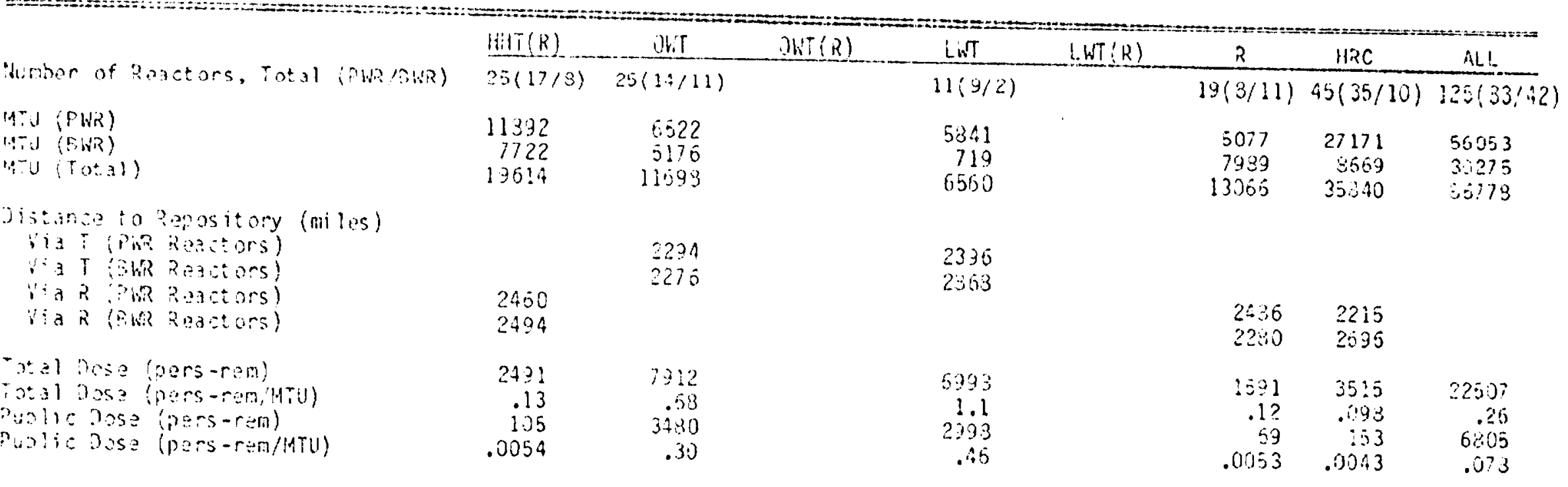

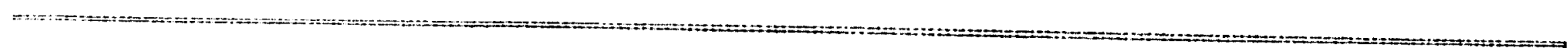


Taule 5-3. LCO Surmury - Case VIlo

Cask Transport Via HHT(R)/OWT(R)/LWT/R/HRC

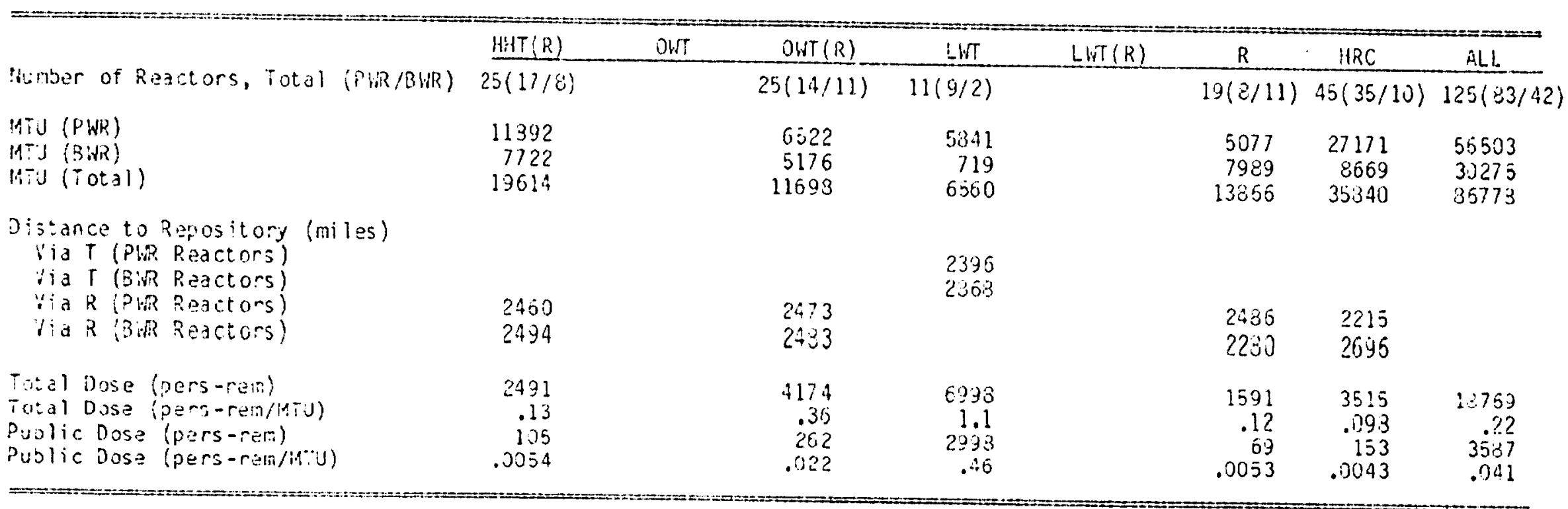


Table 5-9. I.co Sumary - Caso VIIc

Cask Transport Via Hi:T(R)/OWT(R)/LWT(R)!R/HPC.

\begin{tabular}{|c|c|c|c|c|c|c|c|c|}
\hline & $\{\{1 !(P\}$ & bit & $J H(R)$ & $16 \pi$ & $\operatorname{LIV}(k)$ & $R$ & 40 & $\therefore L$ \\
\hline 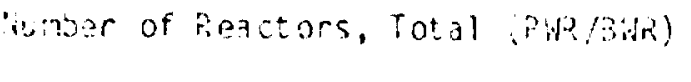 & $25(17 / 3)$ & & $25(14 / 11)$ & & $11(9 / 2)$ & $19(3 / 11)$ & $45(25,10)$ & $125(23,42)$ \\
\hline 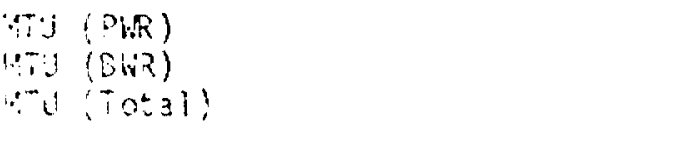 & $\begin{array}{r}11392 \\
772 ? \\
17626\end{array}$ & & $\begin{array}{r}5522 \\
5176 \\
11543\end{array}$ & & $\begin{array}{r}5811 \\
719 \\
6550\end{array}$ & $\begin{array}{r}5077 \\
7939 \\
1 ? 756\end{array}$ & $\begin{array}{r}27171 \\
3569 \\
33640\end{array}$ & $\begin{array}{l}36593 \\
31235 \\
05173\end{array}$ \\
\hline 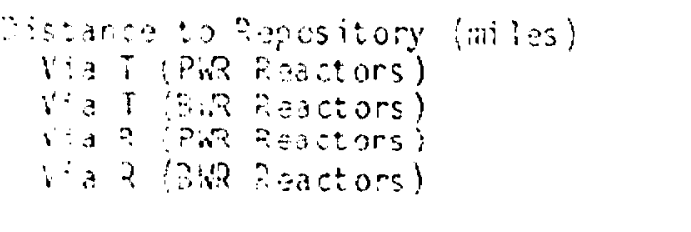 & $\begin{array}{l}3000 \\
2404\end{array}$ & & $\begin{array}{l}2473 \\
2493\end{array}$ & & $\begin{array}{l}2525 \\
3129\end{array}$ & $\begin{array}{l}2435 \\
2230\end{array}$ & $\begin{array}{l}2 ? 15 \\
2596\end{array}$ & \\
\hline 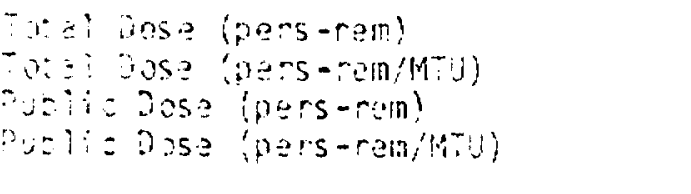 & $\begin{array}{r}241 \\
.13 \\
105 \\
0.954\end{array}$ & & $\begin{array}{r}.174 \\
.36 \\
252 \\
.022\end{array}$ & & $\begin{array}{r}3773 \\
.63 \\
25.1 \\
.027\end{array}$ & $\begin{array}{r}1591 \\
.1 ? \\
07 \\
.1053\end{array}$ & $\begin{array}{r}2515 \\
.993 \\
153 \\
.0713\end{array}$ & $\begin{array}{r}155.43 \\
.13 \\
243 \\
.019\end{array}$ \\
\hline
\end{tabular}




\subsection{SUHHARY AND CONCLUSIONS}

Life-cycle cost (LCC) and life-cycle dose (LCD) estimates have been made for seven transport modal options and three subcases pertaining to the "No-New Orders-'85" nuclear waste scenario. The No-'86 scenario encompasses 125 reactor plants and waste pools. The results of these estimates are summarized in Table 6-1 and shown graphically in Figure 6-1.

Table 0-1. Sumary LCC/LCD Estimates

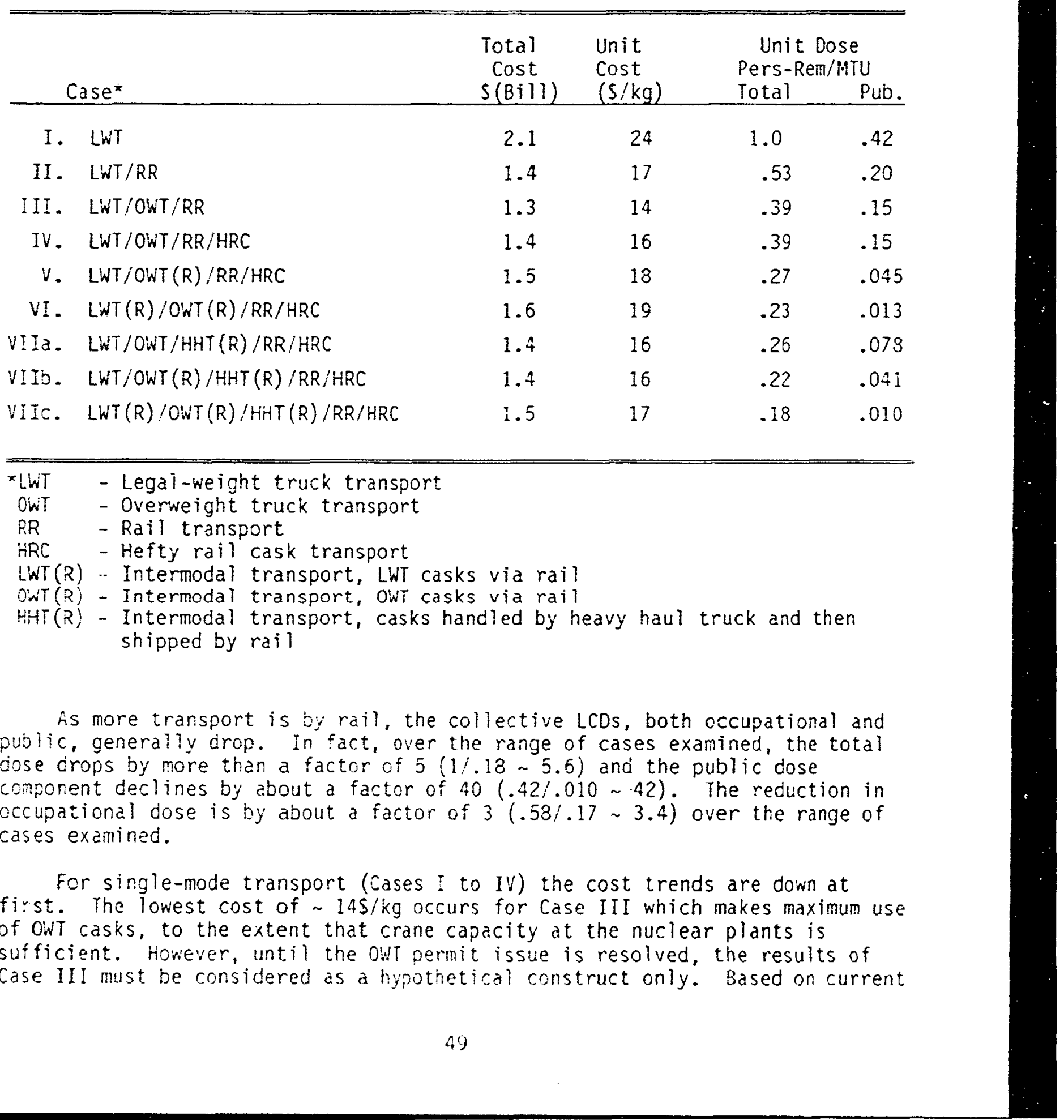


cost data, truck transport is considera'ly more expensive than rail transport. Hefty railcar transport tends to be more expensive, mostly because dedicated rail charges on the empty cask return trip are collected, which is not the case for regular rail cask transport. If hefty rail casks were assumed to be returned by regular freight, then the use of hefty rail casks would result in costs essentially the same as for regular rail casks.

The intermodal transport schemes (Cases $V$ to VII) all involve more handling and transport time than single-mode transport and the corresponding shipping costs increase.

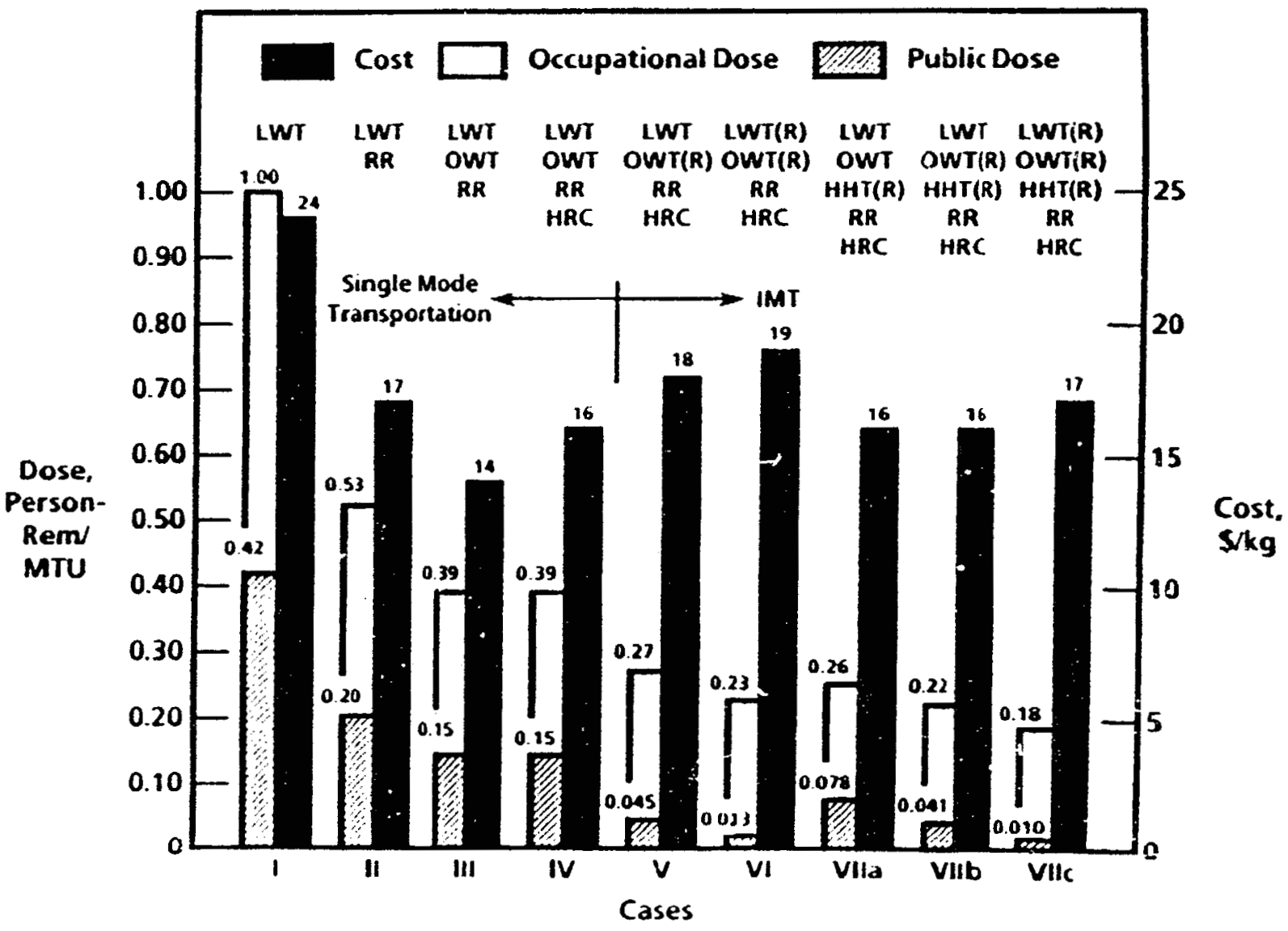

Figure 6-1. LCC/LCD Summary 


\subsection{REFERENCES}

(1) U.S. Department of Energy, 1988. Transfortation Institutional Plan, OOE/Ris-0094, Office of Civilian Radioactive Waste Management, Washington, O.C., p. A-88, August.

(2) U.S. Department of Energy, 1988. Systems Engineering Management Plan, COE/R!:-005:, Office of Civilian Radioactive Waste Management, Washington, D.C., P. 14, October.

(3) U.S. Department of Energy, 1987. Standard Contract for Disposal of Spent Nuclear Fuel and/or High-Levei Radioactive Waste, January 1, 1987, 10 CFR Part 961.

(4) Energy Information Administration, 1987. Commercial Nuclear Power 1987 Prospects for the United States and the World, COE/EIÁ-0438(87), Washington, D.C.

(5) Walling, R. C., C. M. Heeb, and W. L. Purcell, 1988. Reactor-Specific Spent Fuel Discharge Projections 1987 to 2020, PNL-6430, Pacific Northwest Laboratory, Richland, WA, March.

(5) Peterson, B. E., 1983. INTERLINE, A Railroad Routing Mode 1: Program Description and User's Manual, ORNL/TH-3944, Oak Ridge National Laboratory, Oak Ridge, TN.

(7) Joy, D. S., P. E. Johnson, and S. H. Gibson, 1982. HIGHWAY, A Transportation Routing Model: Program Description and User's Manual, ORNL/TM-8149, Oak Ridje National Laboratory, Oak Ridge, TN.

(8) Pacific Northwest Laboratory, 1987. Analys is of Radiation Doses from Operation of Postulated Commercial Spent Fuel Transportation Systems, DOE/CH/TPO-CO1, prepared for U.S. Department of Energy, November.

(9) Dippold, D. G., R. W. Peterson, and L. A. Brentlinger, 1987. "Analyzing Nuclear Waste Transportation Options: The Use of Life Cycle Cost, "J. Q. Chou, ed. Proceedings of the 1927 Summer Computer simulation Conference (July 27-30, Queber, Canada), pp. 1013-1017.

(10) Madsen, Marcella M., John M. Tayior, Robert il. Ostmeyer, and Philip C. Reardon, 1986. RÁDTRAil III, SAND84-0036, Sandia National Laboratories, Albuquerque, NH.

(11) Anderson, R. T., et al.. February 1978. Current Status and Future Considerations for a Transportation System for Spent Fuel and Radioactive iaste, Y/Oni/SUB-77-42513, prepared by Allied-General Nuclear Services for Office of Waste Isolation, U.S. Department of Energy, Oak Ridge, IN.

(12) Unione, A. J., et al., September 1972. A Generic Assessment of Barge Transportation of Spent Nuclear Fuel, AIF/NES?-014, prepared for Atomic Industrial Forum by science fpplications International, Inc. 
(13) Gray, John E., 1976. Nuclear Fuels Policy, Report of the Atlantic Council's Nuclear Fuels Policy Working Group, Atlantic Council of the United States.

(14) Voilieque, P. G. Septenter 1932 . "Socieial Cost of Radiation Exposure," Health Physics, Vol. 43, No. 3 (September), pp. 405-409. Printed in U.S.S. 


\section{APPENDIX $A$}

ICC MODEL DESCRIPTION

The life-cycle costs (LCCS) corresponding to the legal-weight truck (LWT) transport of the $3 / 7$ and $4 / 9$ cask designs were calculated using the computer code CASKCOM, modified to include current transportation assumptions.

The objective of the cask LCC model is to estimate the total costs of handling and transporting a fleet of shipping cask designed to move some given quantity of PWR and BWR spent nuclear fuel. The following cost components of spent fuel transportation are estimated:

- Development and certification cost

- Cask acquisition cost

- Reactor handling cost

- Cask maintenance cost

- Hauling cost

- Security cost

- Repository handling cost.

\section{A.1 MODEL ASSUMPTIONS}

Certain simplifying assumptions are incorporated into the LCC model. The major assumptions include:

- Spent fuel is shipped between one origin and one destination which is linked by a fixed distance. This distance represents the weighted-average distance from each reactor, in a specified set of reactors, to the Nevada Yucca Mountain site, where the MTU discharges of spent fuel serve as weighting factors in calculating the distance.

- Average speed of the shipment while en route is fixed. In the case of truck transport, the average speed is based on the origindestination distance and the cask size being utilized.

- Cask fleet size is calculated as a function of the total cask turnaround time and cask utilization, where turnaround time is a composite of turnaround time at the reactor and at the repository plus the round-trip in-transit time.

- Cask life is estimated to be 20 years.

- Annual quantity of waste shipped is fixed and is based on the specified set of reactors with their corresponding MTU spent fuel discharges through life.

- Costs are tabulated in constant 1987 dollars, and it is assumed that there is no cost escalation. 


\section{A.2 MODEL LOGIC}

What follows is a description of the individual cost calculations that are pertinent to legal weight truck transport and are contained in the current CASKCOM code.

\section{A.2.1 Development and Certification Costs}

The cask development and certification costs depend upon the nature of the cask design and the degree to which it departs from traditional concepts. An estimate of the total development and certification costs is distributed over time using whatever distribution is deemed appropriate.

$$
\begin{gathered}
\text { DCERT(IYEAR) }=\text { DAC*DCDIST(IYEAR) } \\
\text { where DAC is the total development and certification } \\
\text { COSt, and } \\
\text { DCOIST (IYEAR) is the percentage of DAC accruing } \\
\text { to year IYEAR. }
\end{gathered}
$$

\section{A.2.2 Acquisition Cost}

Acquisition cost is not simply the cost of purchasing one cask. Because such cask design features as utilization rate and turnaround time affect the effective capacity of a cask, it is necessary to base acquisition cost on the fleet size required to transport a fixed quantity of spent fuel between a given origin and destination within a given time period. The time required for a cask to complete a round trip is estimated as

$$
\begin{aligned}
\operatorname{TTIME}(W T)= & \operatorname{DIST}(1 / \text { SPEEDL/24) }+ \text { DIST }(1 / \text { SPEEDE } / 24)+ \\
& \operatorname{TURN}(W T, R)+\operatorname{TURN}(W T, R E P)+\operatorname{TRANSF}(W T)
\end{aligned}
$$

$$
\begin{aligned}
& \text { where DIST is the one-way distance setween origin and } \\
& \text { destination, } \\
& \text { SPEEDL is the average speed on the loaded front haul, } \\
& \text { SPEEDE is the average speed on the empty back haul, } \\
& \text { TURN(WT, R) is the average number of days required to } \\
& \text { handle cask of type WT at the reactor (calculated } \\
& \text { aS a function of shipment size), } \\
& \text { TURN(WT, REP) is the number of days required to handle } \\
& \text { a CaSk of type WT at the repository, } \\
& \text { TRANS (WT) is the number of days required to } \\
& \text { transfer a cask of type WT from one transport mode } \\
& \text { to another, and } \\
& \text { WT is an index referring to either PWR or BWR. }
\end{aligned}
$$

If the transport mode is rail, then the average turnaround time per cask at the reactor is calculated as a function of shipment size to be

$$
\operatorname{TUPN}(W T, R)=[((N \star * 2+N) / 2) * \text { CASTUPN }(W T, R)] / N
$$


where $N$ is the number of casks per train shipment, and CASTURN (WT, $R$ ) is the number of days required to handle a cask of type WT.

If the transport mode is truck, then average turnaround time at reactor per cask TURN(WT; is assumed equal to the estimated turnaround time per cask CASTURN(WT) because it is assumed that truck casks are dispatched singly from the reactor.

The annual number of trips that each PwR/BNR cask is able to make is

$$
\begin{aligned}
& \text { ATRIP(WT) = DAYS*UTIL(WT)/TMIME (WT) } \\
& \text { where DAYS are the number of days per year, and } \\
& \text { UTIL(WT) is the percentage of time that the cask } \\
& \text { of type WT is operational. }
\end{aligned}
$$

The amount of spent fuel that each type of cask is able to carry annually is a product of the annual trips per cask and the capacity of the cask, such that

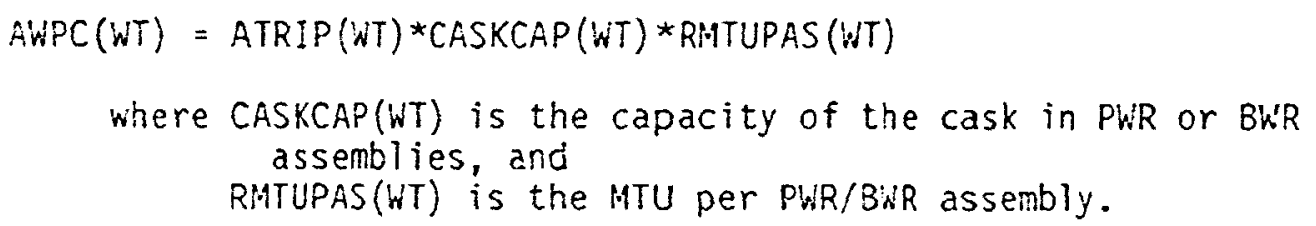

The cask fleet needed to transport the total amount of PWR and BWR spent fuel from the origin to the destination is

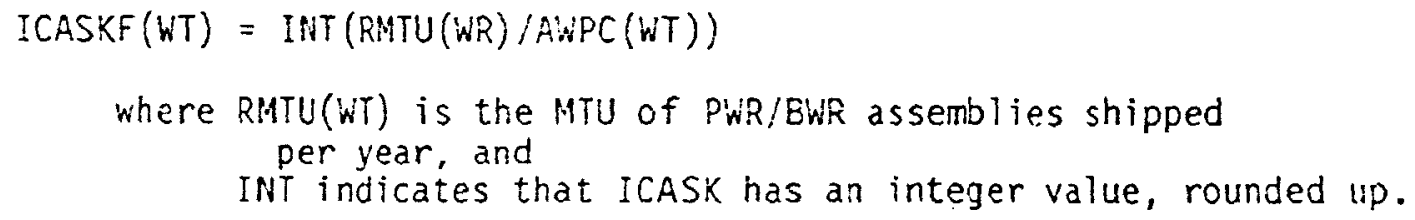

The total cask fleet needed to transport both the. Pir and BWR spent fuel is the sum of the two cask fleets.

$$
\text { FLEETTOT = ICASKF (PWR) + ICASKF (BWR) }
$$

Because the possibility exists that there are scale effects associated with the cost of fabricating the cask fleet, the cost per cask is a function of the size of the total cask fleet (PUP plus BWR). In order to capture this scale effect, the total cask fleet acquisition cost is interpolated from data provided on the cost of fabricating cask fleets of 10,20 , and 70 casks, respectively. The cost of acquiring a cask fleet of size fLEETTOT is then interpolated from these design data. 


\section{CCOST = INTERPOLATION [FLEETTOT, CASK DESIGNERS' COST DATA] \\ where INTERPOLATION indicates that the value of CCOST is interpolated using FLEETTOT and cask design data from the cask designers.}

The number of years required to fabricate the cask fleet is interpolated from data previously provided by various cask designers and is then used to distribute the acquisition costs uniformly over that time period such that

$$
\operatorname{CAPCOST}(Y E A R)=\operatorname{COOST} / N Y R
$$

\section{A.2.3 Operating Costs}

The annual fleet operating costs include the annual hauling costs, security costs, maintenance costs, and handling costs. The annual handling costs for the PWR/B'R casks are

$$
\begin{aligned}
& \text { HAULC (WT) }=\text { (WTE (WT)*EMPTYC + WTL (WT)*LOADC) } \\
& \text { *ICASKF (WT)*ATRIP(WT) } \\
& \text { where WTE(WT) is the empty weight (in hundredweight CWT) } \\
& \text { Of the PWR/BWR cask, and } \\
& \text { WTL(WT) is the loaded weight (CWT) of the cask. }
\end{aligned}
$$

The unit hauling costs for an empty and loaded cask, EMPTYC and LOADC, depend upon the mode of cask shipment. For truck transport the unit hauling costs are

$$
\begin{aligned}
& \text { EMPTYC }=.00402 * \text { DIST }+.3954 \\
& \text { LOADC }=.004764 * \text { DIST }+1.1614
\end{aligned}
$$

For rail transport the unit hauling costs are estimated by

$$
\begin{aligned}
& \text { EMPTYC }=.03636 * \text { DIST } \star * .586 \\
& \text { LOADC }=.03636 \star \text { DIST } \star .586
\end{aligned}
$$

when a dedicated train is assumed, then an additional per train-mile charge of $\$ 48$ is used.

The average annual cask security cost for truck transport is calculated as a function of distance to be 


$$
\operatorname{SECCOST}(W T)=\mathrm{DIST} * 3.688
$$

For rail transport, the cak security cost is calculated as

$$
\begin{aligned}
\operatorname{SECCOST}(W T)= & (.76 * D I S T)+500 \text { (DIST/SPEEDL + DIST/SPEEDE) } \\
& +500 * \operatorname{CASTURN}(W T, R)
\end{aligned}
$$
BWR cask.

Annual fleet maintenance costs depend upon whether the cask is a PWR or

$$
\begin{aligned}
& \text { CMAINT }(W T)=\operatorname{ICASKF(WT)~} \times \operatorname{UCM}(W T) \\
& \text { where UCM }(W T) \text { is the annual cost of maintaining } \\
& \text { a PWR/BWR cask. }
\end{aligned}
$$

The annual cost of handling the PWR/BWR cask fleet at the reactor and the repository is

$$
\begin{aligned}
& \text { HANDC (WT) }=\left(P R O C(W T, R){ }^{\star} R C O S T(W T) \star C R E W(R)+P R O C(W T, R E P)\right. \\
& \left.\star R E P C O S T(W T)^{\star} C R E W(R E P)\right)^{\star} A T R I P(W T) \star I C A S K F(W T) \\
& \text { where } P R O C(W T, R) \text { is the time required to process the } \\
& \text { PWR/BWR Cask at the reactor, } \\
& \text { PROC(WT, REP) is the time required to process the } \\
& \text { PWR/BWR cask at the repository, } \\
& \text { RCOST(WT) is the hourly cost of handling the PWR/BWR } \\
& \text { CaSk at the reactor, } \\
& \text { REPCOST(WT) is the hourly cost of handling the } \\
& \text { PWR/BWR at the repository, and } \\
& \text { CREW(R) and CREW(REP) are the crew sizes at the } \\
& \text { reactor and repository, respectively. }
\end{aligned}
$$

The total annual fleet operating cost is, therefore, the sum of annual hauling, security, maintenance, and handling costs for both the PWR and BWR casks.

$$
\begin{gathered}
\text { TOTOPER = SUM(WT) [HAULC (WT) + SECCOST (WT) + MAINTC (WT) + HANDC (WT)] } \\
\text { where SUM(WT) indicates that the variables in the } \\
\text { brackets are summed for both PWR and BWR. }
\end{gathered}
$$

